\title{
Preferences for Flexibility and Randomization under Uncertainty
}

\author{
By Kota SaIto*
}

\begin{abstract}
An uncertainty-averse agent prefers betting on an event whose probability is known, to betting on an event whose probability is unknown. Such an agent may randomize his choices to eliminate the effects of uncertainty. For what sort of preferences does a randomization eliminate the effects of uncertainty? To answer this question, we investigate an agent's preferences over sets of acts. We axiomatize a utility function, through which we can identify the agent's subjective belief that a randomization eliminates the effects of uncertainty. (JEL D11, D81)
\end{abstract}

People often prefer to bet on a risky event (an event whose probability is known) rather than an uncertain event (an event whose probability is unknown). Such a preference is said to be uncertainty-averse. (See Gilboa 2009 for a formal definition of uncertainty aversion.) If you have such a preference, one way to make a choice is to toss a coin.

For example, consider a bet on which country, A or B, will win the final match of FIFA World Cup. If your bet turns out to be correct, you obtain $\$ 100$; otherwise you obtain $\$ 0$. The problem is that you do not necessarily have a probabilistic assessment on which country will win. Suppose that you toss a fair coin and you bet on A if heads appears; otherwise, you bet on B. Then, no matter which country wins, you can obtain the fifty-fifty lottery between $\$ 100$ and $\$ 0$.

Yet, are you really indifferent between tossing a fair coin and obtaining the fifty-fifty lottery between $\$ 100$ and $\$ 0$ ? In other words, do you really think the coin toss eliminates the effects of uncertainty?

This is just one example of many economic decisions under uncertainty. For what sort of preferences does a randomization eliminate the effects of uncertainty? To answer the question, we investigate an agent's preferences over sets of alternatives.

\footnotetext{
* Division of the Humanities and Social Sciences, California Institute of Technology, MC 228-77, 1200 E. California Blvd., Pasadena, CA 91125 (e-mail: saito@caltech.edu). I am indebted to my adviser, Eddie Dekel, for continuous guidance, support, and encouragement. I would like to thank Federico Echenique for continuous discussion that has led to improvement of the paper. I am grateful to Larry Epstein, Jeff Ely, Faruk Gul, Edi Karni, Michihiro Kandori, Pietro Ortoleva, Fabio Maccheroni, Massimo Marinacci, Wolfgang Pesendorfer, Kyoungwon Seo, Chris Shannon, Marciano Siniscalchi, Peter Wakker, Leeat Yariv, and seminar participants at the Michigan University, Econometric Society Meeting 2012 at Northwestern University, the Decision Theory Workshop 2012 at the New School of Economics, and RUD 2013 (Paris) for discussions which have led to improvement of the paper. Finally, I appreciate three anonymous referees for their helpful comments. The author declares that I have no relevant or material financial interests that relate to the research described in the paper.

${ }^{\dagger}$ Go to http://dx.doi.org/10.1257/aer.20131030 to visit the article page for additional materials and author disclosure statement(s).
} 
We axiomatize a utility function, through which we can identify the agent's subjective belief that his randomization eliminates the effects of uncertainty.

In the next section, we review Ellsberg's (1961) paradox and Raiffa's (1961) critique. Then, in Section II, we preview our results.

\section{Ellsberg's (1961) Paradox and Raiffa's (1961) Critique}

To give a concrete example of choice under uncertainty, consider the following hypothetical experiment first described by Ellsberg (1961). Suppose that you confront two urns containing red and black balls. You have the following information: Urn I contains 100 red and black balls, but in a ratio entirely unknown to you; there may be from 0 to 100 red balls. In Urn II, you know that there are exactly 50 red and 50 black balls. We call Urn I the uncertain urn and call Urn II the risky urn, respectively. 1

To bet on a color in an urn will mean that we draw a ball from the urn at random, and that you will receive a payoff of 1 if the drawn ball is the color on which you bet and 0 otherwise. We ask four independent questions, each of which offers two alternatives. Which do you prefer to bet on: (i) red or black in the uncertain urn? (ii) red or black in the risky urn? (iii) red in the uncertain urn or in the risky urn? (iv) black in the uncertain urn or in the risky urn?

If you are indifferent between red and black in questions (i) and (ii), and you strictly prefer the risky urn to the uncertain urn in questions (iii) and (iv), then your preferences are said to be uncertainty-averse. Many experimental studies have confirmed such uncertainty-averse preferences in lab experiments.

Is it reasonable for many people to strictly prefer the risky urn to the uncertain urn? According to Raiffa (1961), it may not be. By tossing a fair coin, you can eliminate the effects of uncertainty; you can obtain the fifty-fifty lottery between 1 and 0 for each ball color drawn from the uncertain urn. For example, suppose that you bet on red if heads appears and you bet on black if tails appears. Then what you obtain is the fifty-fifty lottery between 1 and 0 for each ball color drawn from the uncertain urn. This is exactly what you obtain by betting on a color in the risky urn. Hence, there is no reason to strictly prefer the risky urn to the uncertain urn. This is the essence of Raiffa's (1961) critique of uncertainty-averse preferences.

Raiffa's (1961) critique has been widely accepted in the field. However, we claim that there are three important problems with his argument. The first problem is that Raiffa's (1961) argument presupposes that you believe that the coin toss eliminates the effects of uncertainty. To see this, remember that Raiffa (1961) evaluates the coin toss for each ball color drawn from the uncertain urn, as shown by the left tree of Figure 1. However, there is another, equally natural way of evaluating the coin toss: evaluate the coin toss for each realization of the coin toss (i.e., heads or tails), as shown by the right tree of Figure 1 .

According to this latter evaluation, you have to face uncertainty again, no matter which side of the coin appears. The coin toss therefore does not eliminate the effects of uncertainty. Indeed, this latter view of randomization would make more sense if

\footnotetext{
${ }^{1}$ Note that a red (black) ball drawn from Urn I is an uncertain event, while a red (black) ball drawn from Urn II is a risky event.
} 

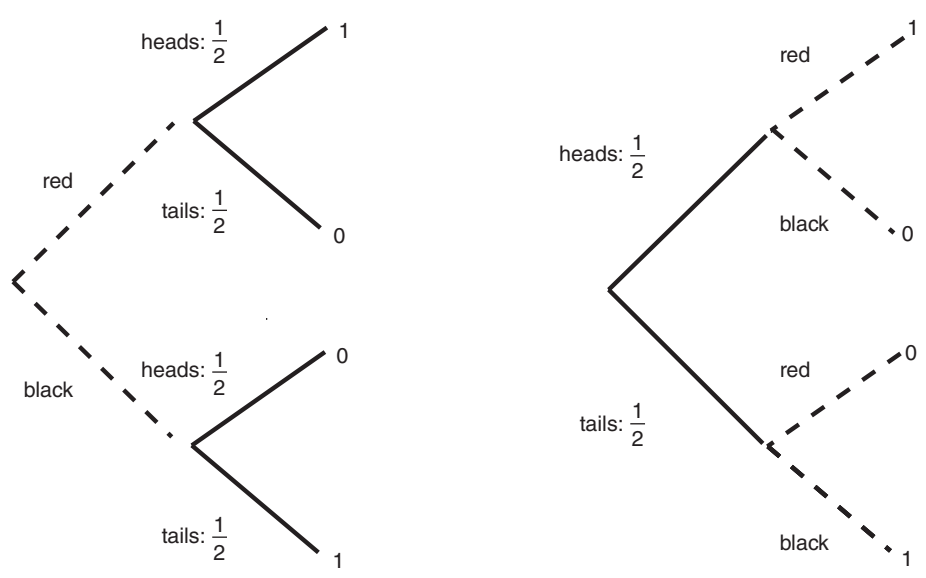

Figure 1. Two Ways to Evaluate the Coin Toss

Note: The coin toss eliminates the effects of uncertainty (left); does not eliminate the effects of uncertainty (right).

you think that a color of the drawn ball is realized after you toss the coin. These two ways of evaluating the coin toss (i.e., evaluating it for each ball color drawn from the uncertain urn or evaluating it for each realization of the coin toss) reflect whether or not you believe that the coin toss eliminates the effects of uncertainty. Both beliefs are legitimate, and which one is correct depends on you.

The second problem with Raiffa's (1961) critique concerns the size of the choice set. Ellsberg (1961, p. 651, footnote 7) identifies this problem by saying: "Note that in no case are you invited to choose both a color and an urn freely; nor are you given any indication beforehand as to the full set of gambles that will be offered." In the four questions, when you are asked your preference between the uncertain urn and the risky urn (namely, in questions (iii) and (iv)), your choice of a color is predetermined so that you cannot randomize between betting on red and betting on black.

The third problem is that Raiffa (1961) has assumed that you can commit to the result of his randomization. To see this point, assume that you slightly prefer betting on red to betting on black. If the coin toss determines betting on black, then you might not be able to commit to the result of the coin toss. ${ }^{2}$

\section{Preview of Results}

Given the discussion above, a natural question to ask is: for what sort of preferences does a randomization eliminate the effects of uncertainty? To study this question, we consider a set $S$ of states and an agent facing uncertainty about which state $s \in S$ is realized. For example, in Ellsberg's (1961) experiment, $S$ consists of the possible ball color drawn from the uncertain urn (i.e., $S=\{$ Red, Black $\}$ ).

\footnotetext{
${ }^{2}$ I am very grateful to the referee who pointed out this important issue.
} 
We axiomatize the agent's preferences over sets of payoff (utilities) profiles (across the states). We have in mind an agent facing a two-stage decision problem. The agent chooses a set in stage 1 and subsequently selects a payoff profile from that set in stage 2 , maybe by randomization (such as tossing a coin). We do not use the agent's random choice as a primitive because it may not be observable: he may randomize his choices in his mind without using observable randomization devices. Consequently, we do not explicitly model a process of random choice. Instead, we leave the agent's random choice (and his commitment to his random choice) to be part of the interpretation of his utility function. Therefore, the third problem of Raiffa's (1961) critique is moot in our model.

We axiomatize a utility function that identifies the agent's subjective belief that his randomization eliminates the effects of uncertainty. We call the utility representation a random uncertainty-averse (RUA) representation. An RUA representation is characterized by a pair $(\delta, C)$ of $\delta \in[0,1]$ and $C \subset \Delta(S)$. The utility of a set $A$ of payoff profiles is

$$
U(A)=\max _{\rho \in \Delta(A)}\left[\delta u\left(\sum_{f \in A} \rho(f) f\right)+(1-\delta) \sum_{f \in A} \rho(f) u(f)\right]
$$

where $u(f)=\min _{p \in C} \sum_{s \in S} p(s) f(s)$, and $\Delta(A)$ is the set of probability distributions over payoff profiles in $A$.

The function $u$ captures uncertainty aversion and corresponds to the maxmin expected utility function proposed by Gilboa and Schmeidler (1989). The parameter $\delta \in[0,1]$ captures the agent's subjective belief that his randomization $\rho$ eliminates the effects of uncertainty. Moreover, the maximizer $\rho$ over $\Delta(A)$ captures the agent's optimal random choice over $A$. In an online Appendix, we present an axiomatization of an extended representation, in which $u$ is a variational utility function proposed by Maccheroni, Marinacci, and Rustichini (2006).

To understand these interpretations of $\delta$ and $\rho$, note that in the objective function, the first term, $u\left(\sum_{f \in \mathscr{F}} \rho(f) f\right)$, is the utility when the randomization $\rho$ eliminates the effects of uncertainty because $\sum_{f \in \mathscr{F}} \rho(f) f$ denotes a state-wise mixture of payoff profiles with respect to $\rho$. For example, in the first term, Raiffa's (1961) coin toss is evaluated as the constant payoff profile $\frac{1}{2}(1,0)+\frac{1}{2}(0,1) \equiv\left(\frac{1}{2}, \frac{1}{2}\right)$, where the payoff profiles $(1,0)$ and $(0,1)$ correspond to betting on red and black, respectively, in the uncertain urn.

On the other hand, the second term, $\sum_{f \in \mathscr{F}} \rho(f) u(f)$, is the utility when the randomization $\rho$ does not eliminate the effects of uncertainty. In this case, the agent evaluates each payoff profile separately and then calculates the expected value with respect to $\rho$. For example, in the second term, the utility of the coin toss is the expected utility of $\frac{1}{2} u(1,0)+\frac{1}{2} u(0,1)$.

Therefore, the parameter $\delta$ captures the agent's subjective belief that his randomization $\rho$ eliminates the effects of uncertainty. In this way, we address the first problem with Raiffa's (1961) critique, namely that Raiffa (1961) presupposes that the agent believes that his randomization eliminates the effects of uncertainty. (In other words, Raiffa 1961 presupposes $\delta=1$.) In our model, the weighted sum of the two terms, $\delta u\left(\sum_{f \in \mathscr{F}} \rho(f) f\right)+(1-\delta) \sum_{f \in \mathscr{F}} \rho(f) u(f)$, is the agent's expected utility 
obtained by his randomization $\rho$. Therefore, the maximizer $\rho$ over $\Delta(A)$ captures the agent's optimal random choice over $A$. Note that we obtain the optimal random choice endogenously, in the sense that our primitive does not include a choice of randomization.

We apply our result to Ellsberg's (1961) experiment to address the second problem with Raiffa's (1961) critique, namely that whether an agent can randomize his choices or not depends on how large his choice set is. We consider an agent whose preferences admit an RUA representation and who faces the two urns described in Section I. Then, we study how the size of the agent's choice set affects his observable attitude toward uncertainty. We show that the agent's uncertainty premium depends on who determines the ball color drawn from the uncertain urn on which he bets. The uncertain premium is lower when the agent determines it than when the experimenter predetermines it. Moreover, we also show that when the agent determines the ball color, his uncertainty premium decreases as $\delta$ increases. In fact, the agent's uncertainty premium becomes zero when $\delta=1$, even when he is uncertainty-averse. See Section V.

We present comparative statics on $\delta$. We show that both a preference for randomization and a preference for flexibility (i.e., preference for a larger set) are captured in a natural way by $\delta$. See Section IV. All proofs are in Section VI.

Finally, let us now discuss the related literature. Our paper is related to papers on subjective state space, such as the work of Dekel, Lipman, and Rustichini (2001) and Epstein, Marinacci, and Seo (2007). Epstein, Marinacci, and Seo (2007) point out that an agent can eliminate the effects of uncertainty by randomizing his choice; they then axiomatize two models that are similar to the two special cases of RUA representations with $\delta=1$ and $\delta=0$. Our paper, however, is different from both papers in its motivations and primitives. The main purpose of Dekel, Lipman, and Rustichini (2001) and Epstein, Marinacci, and Seo (2007) is to obtain a state space endogenously, a space which has often been assumed as a primitive (e.g., in Savage 1954 and Anscombe and Aumann 1963 as well as in our paper). Consequently, the primitive preferences of Dekel, Lipman, and Rustichini (2001) and Epstein, Marinacci, and Seo (2007) are defined on sets of payoffs (i.e., lotteries), while our primitive preferences are defined on sets of payoff profiles across the state space.

Our paper is also related to recent axiomatic literature on random choice, such as Battigalli et al. (2013), Gul and Pesendorfer (2006), and Manzini and Mariotti (2014). Their primitives and motivations are also different from ours. They all use random choice functions as primitives. Moreover, only Battigalli et al. (2013) study uncertainty-averse agents. The main purpose of Battigalli et al. (2013), however, is different from ours in that they present a new framework for studying random choice under uncertainty, while maintaining the mathematical convenience of Anscombe and Aumann's (1963) framework.

In Saito (2013), we have also studied a preference for randomization. There, we focus on other-regarding behaviors in a social context, such as when two agents toss a coin to decide who gets an indivisible good. In contrast to the present paper, the primitive preferences in that paper are defined on randomizations over payoff profiles (across the agents). This difference in primitives arises from the fact that randomizations would be observable in a social context. For example, the two agents 
would use observable randomization devices to decide who gets the indivisible good. Because of the difference in primitives, our proof here is completely different from the proof in Saito (2013).

There is recent research on a preference for randomization under uncertainty, in which primitive preferences are defined on randomizations over payoff profiles (across the state space). Eichberger, Grant, and Kelsey (2014) show that a dynamically consistent agent has no preference for randomization. Kuzmics (2013) shows that if an agent can randomize his choice in his mind, he can commit to his randomization, and if he believes that his randomization eliminates the effects of uncertainty, then he behaves as if uncertainty-neutral.

\section{Axioms}

We denote the set of payoffs (i.e., von Neumann-Morgenstern utilities) by $\mathcal{U}$. We assume that $\mathcal{U}$ is a nonempty, compact, and convex subset of real numbers. ${ }^{3} \mathrm{We}$ also assume that $\mathcal{U}$ is not a singleton. ${ }^{4}$ We denote the set of real numbers by $\mathbb{R}$ and the set of nonnegative real numbers by $\mathbb{R}_{+}$. Let $S=\{1, \ldots, n\}$ be the set of states. A payoff profile $f=(f(1), \ldots, f(n)) \in \mathcal{U}^{S}$ is called an act. We denote the set $\mathcal{U}^{S}$ of acts by $\mathscr{F}$. Let $\mathscr{A}$ be the set of all nonempty compact subsets of $\mathscr{F}$. A probability distribution $\rho$ over acts with finite support is called a randomization. For any set $A \in \mathscr{A}$, we denote by $\Delta(A)$ the set of randomizations over acts in $A$.

The primitive of our model is a binary relation $\succsim$ on $\mathscr{A}$, which describes the agent's choice of sets..$^{5}$ Note that this domain of $\succsim$ does not include randomizations (i.e., $\Delta(\mathscr{F}) \not \subset \mathscr{A})$. In this sense, we do not assume that the agent's choices over randomizations are observable.

An act $f$ is called a constant act if $f(s)=f\left(s^{\prime}\right)$ for all $s, s^{\prime} \in S$. For an act $f$, a payoff $x \in \mathcal{U}$ is called the certainty equivalent of $f$, if $f \sim(x, \ldots, x)$. We denote the certainty equivalent of $f$ by $x(f)$.

Payoffs are denoted by $x, y \in \mathcal{U}$. Sets of acts are denoted by $A, B \in \mathscr{A}$. Acts are denoted by $f, g, h \in \mathscr{F}$. Randomizations are denoted by $\rho, \mu \in \Delta(\mathscr{F})$. If $\rho$ is a randomization that yields act $f^{i}$ with probability $\rho_{i} \in[0,1]$ for each $i \in\{1, \ldots, m\}$, then we write $\rho=\rho_{1} f^{1} \oplus \cdots \oplus \rho_{m} f^{m}$.

We define state-wise mixtures of acts and sets as follows:

DEFINITION 1: For all $\alpha \in[0,1]$ and $f, g \in \mathscr{F}, \alpha f+(1-\alpha) g$ is an act such that $(\alpha f+(1-\alpha) g)(s)=\alpha f(s)+(1-\alpha) g(s) \in \mathcal{U}$ for each $s \in S$.

For any $f^{1}, \ldots, f^{n} \in \mathscr{F}$ and any $\alpha_{1}, \ldots, \alpha_{n} \in[0,1]$ such that $\sum_{i=1}^{n} \alpha_{i}=1$, we denote $\alpha_{1} f^{1}+\cdots+\alpha_{n} f^{n}$ by $\sum_{i=1}^{n} \alpha_{i} f^{i}$. In the following, by mixing acts, we mean making a state-wise mixture among the acts. Note that the state-wise mixture of acts is not a (nondegenerate) randomization.

\footnotetext{
${ }^{3}$ With a set $Z$ of outcomes, we can use the standard domain $\Delta(Z)$ instead of $\mathscr{U}$ without any major changes in results.

${ }^{4}$ I am very grateful to the referee who suggested this assumption.

${ }^{5}$ As usual, $\succ$ and $\sim$ denote the asymmetric and symmetric parts of $\succsim$, respectively.
} 
DEFINITION 2: For all $\alpha \in[0,1]$ and $A, B \in \mathscr{A}, \quad \alpha A+(1-\alpha) B$ $=\{\alpha f+(1-\alpha) g \mid f \in A$ and $g \in B\}$.

The first five axioms are based on the axioms in Gilboa and Schmeidler (1989).

Axiom (Weak Order): $\succsim$ is complete and transitive.

Axiom (Continuity): For any $f, g \in \mathscr{F}$ and $A \in \mathscr{A}$, if $\{f\} \succ A \succ\{g\}$ then $\alpha\{f\}+(1-\alpha) A \succ A \succ \beta\{g\}+(1-\beta) A$ for some $\alpha$ and $\beta$ in $(0,1)$.

Axiom (Monotonicity): For any $f, g \in \mathscr{F}$, if $f(s) \geq g(s)$ for all $s \in S$ then $\{f\} \succsim\{g\}$. Moreover, if $f(s)>g(s)$ for all $s \in S$ then $\{f\} \succ\{g\}$.

Since $\mathcal{U}$ is neither empty nor singleton, there exist $x, y \in \mathcal{U}$ such that $x>y$. Hence, the monotonicity axiom implies that $(x, \ldots, x) \succ(y, \ldots, y)$. So, $\succ$ is nondegenerate.

The next and last axiom captures the agent's uncertainty aversion. To motivate the axiom, consider the two urns in Section I. Hence, the state space consists of the possible ball color drawn from the uncertain urn (i.e., $S=\{$ Red, Black $\}$ ). The acts $f^{\text {Red }} \equiv(1,0)$ and $f^{\text {Black }} \equiv(0,1)$ correspond to betting on red and black, respectively, in the uncertain urn. The act $\left(\frac{1}{2}, \frac{1}{2}\right)$ corresponds to betting on a color in the risky urn; this is because by doing so, the agent can obtain the expected payoff of $\frac{1}{2}$ independent of a realization of a state. The uncertainty-averse agent prefers $\left(\frac{1}{2}, \frac{1}{2}\right) \equiv \frac{1}{2} f^{\text {Red }}+\frac{1}{2} f^{\text {Black }}$ to $f^{\text {Red }}$ and $f^{\text {Black }}$, if he is indifferent between $f^{\text {Red }}$ and

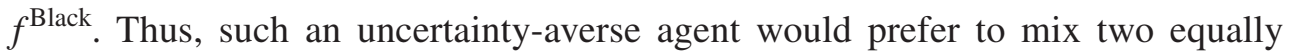
desirable acts, because mixing nonconstant acts can make the mixed payoff profile smoother.

Axiom (Uncertainty Aversion): For any $f, g \in \mathscr{F}$, if $\{f\} \sim\{g\}$ then $\left\{\frac{1}{2} f+\frac{1}{2} g\right\} \succsim\{f\}$.

On the other hand, mixing an act with a constant act might not make the mixed payoff profile smoother. Hence, such a mixture does not reverse the agent's preference between two sets. This observation motivates the following axiom, which is a natural extension of Gilboa and Schmeidler's (1989) certainty independence axiom. 6

Axiom (Certainty Set Independence): For any $x \in \mathscr{U}, A, B \in \mathscr{A}$, and $\alpha \in[0,1]$,

$$
A \succsim B \Leftrightarrow \alpha A+(1-\alpha)\{(x, \ldots, x)\} \succsim \alpha B+(1-\alpha)\{(x, \ldots, x)\}
$$

\footnotetext{
${ }^{6}$ Gilboa and Schmeidler's (1989) certainty independence axiom is as follows: $f \succsim g \Leftrightarrow \alpha f+(1-\alpha)$ $\times(x, \ldots, x) \succsim \alpha g+(1-\alpha)(x, \ldots, x)$.
} 
The next two axioms are new. The first of these, certainty strategic rationality, is significantly weaker than Kreps's (1979) strategic rationality axiom. Kreps (1979) requires that if $A \succsim B$, then $A \sim A \cup B$. The uncertainty-averse agent could violate this axiom. Even if the agent has the option to choose from a set $A$ and he prefers $A$ to another set $B$, he might have a strict preference for the additional option to choose from $B$ so that he could randomize acts in $A$ as well as acts in $B$. However, when $A$ consists of a constant act (i.e., $A=\{(x, \ldots, x)\})$, the agent might not have the strict preference for the additional option because a randomization with the constant act would not make the payoff profile smoother. This suggests the following weaker axiom.

Axiom (Certainty Strategic Rationality): For any $f, g \in \mathscr{F}$ and $x \in \mathcal{U}$,

$$
\{(x, \ldots, x)\} \succsim\{f, g\} \Rightarrow\{(x, \ldots, x)\} \sim\{(x, \ldots, x), f, g\} .
$$

The last axiom is related to Dekel, Lipman, and Rustichini's (2001) indifference to randomization axiom. A natural extension of their axiom to our domain is: $A \sim \operatorname{co}(A)$, where $\operatorname{co}(A)$ denotes the convex hull with respect to the state-wise mixtures (i.e., $\operatorname{co}(A)=\left\{\sum_{i=1}^{n} \alpha_{i} f^{i} \mid f^{1}, \ldots, f^{n} \in \mathscr{F}\right.$ and $\alpha_{1}, \ldots, \alpha_{n} \in[0,1]$ such that $\left.\left.\sum_{i=1}^{n} \alpha_{i}=1\right\}\right) .7$

Whether the agent satisfies this indifference to randomization axiom depends on whether he believes that his randomization eliminates the effects of uncertainty. To see this, consider the two urns in Section I again. Remember that the acts $f^{\text {Red }} \equiv(1,0)$ and $f^{\text {Black }} \equiv(0,1)$ correspond to betting on red and black, respectively, in the uncertain urn; and that the act $\left(\frac{1}{2}, \frac{1}{2}\right)$ corresponds to betting on a color in the risky urn.

Suppose that the agent can determine the ball color drawn from the uncertain urn on which he bets; then his choice set is $\left\{f^{\text {Red }}, f^{\text {Black }}\right\}$. Note that $\operatorname{co}\left(\left\{f^{\text {Red }}, f^{\text {Black }}\right\}\right) \equiv\{(p, 1-p) \mid p \in[0,1]\}$, and the convex hull contains the act $\left(\frac{1}{2}, \frac{1}{2}\right)$. Therefore, the uncertainty-averse agent would strictly prefer $\operatorname{co}\left(\left\{f^{\text {Red }}, f^{\text {Black }}\right\}\right)$ to $\left\{f^{\text {Red }}, f^{\text {Black }}\right\}$ in order to choose $\left(\frac{1}{2}, \frac{1}{2}\right)$, unless he believes what Raiffa (1961) claims, namely that his coin toss eliminates the effects of uncertainty completely.

We propose a new axiom, dominance, which the agent can satisfy independent of his belief. The dominance axiom states that the agent should prefer a set $A$ to another set $B$, if $A$ provides better randomization than $B$, regardless of whether he believes that his randomization eliminates the effects of uncertainty.

To define the dominance axiom, we formalize two ways to evaluate a randomization depending on the agent's belief about the randomization. To illustrate the two ways, consider the two urns in Section I again and note that the coin toss is described as $\frac{1}{2} f^{\text {Red }} \oplus \frac{1}{2} f^{\text {Black }} \equiv \frac{1}{2}(1,0) \oplus \frac{1}{2}(0,1)$. If the agent believes—as Raiffa $(1961)$

\footnotetext{
${ }^{7}$ Dekel, Lipman, and Rustichini (2001, p. 904) have proposed this axiom for preferences over sets of lotteries but not for preferences over sets of acts.
} 

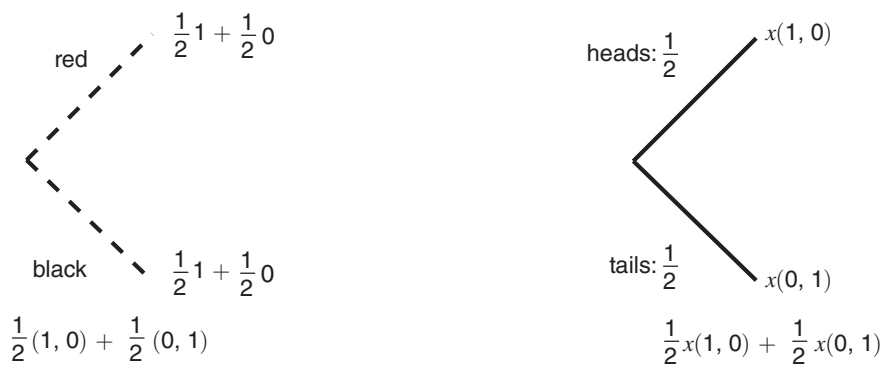

Figure 2. Value of Randomization $\frac{1}{2}(1,0) \oplus \frac{1}{2}(0,1)$ When It Does $($ Left $)$ and Does Not $(R i g h t)$ Eliminate the EFFects of Uncertainty

Note: $x(f)$ denotes the certainty equivalent of act $f$.

claims - that the coin toss eliminates the effects of uncertainty, then he would evaluate the randomization as the state-wise mixture $\frac{1}{2}(1,0)+\frac{1}{2}(0,1) \equiv\left(\frac{1}{2}, \frac{1}{2}\right)$.

On the other hand, if the agent thinks that the coin toss does not eliminate the effects of uncertainty, then he should be indifferent to reducing each act (namely $(1,0)$ and $(0,1))$ to its certainty equivalent (namely $x(1,0)$ and $x(0,1)$ ). Because mixtures among constant acts do not affect uncertainty. Hence, the value of the coin toss should be equal to the expected value $\frac{1}{2} x(1,0)+\frac{1}{2} x(0,1)$ of the certainty equivalents. ${ }^{8}$ These observations suggest the following two ways to compare randomizations that depend on the agent's belief:

DEFINITION 3: For all $\rho, \mu \in \Delta(\mathscr{F})$ such that $\rho=\rho_{1} f^{1} \oplus \cdots \oplus \rho_{m} f^{m}$, $\mu=\mu_{1} g^{1} \oplus \cdots \oplus \mu_{k} g^{k}, \rho$ dominates $\mu$ if

(i) $x\left(\rho_{1} f^{1}+\cdots+\rho_{m} f^{m}\right) \geq x\left(\mu_{1} g^{1}+\cdots+\mu_{k} g^{k}\right)$; and

(ii) $\rho_{1} x\left(f^{1}\right)+\cdots+\rho_{m} x\left(f^{m}\right) \geq \mu_{1} x\left(g^{1}\right)+\cdots+\mu_{k} x\left(g^{k}\right)$.

Definition 3 (i) captures the comparison of randomizations $\rho$ and $\mu$ when the agent believes that the randomizations eliminate the effects of uncertainty. Definition 3 (ii) captures the comparison when the agent believes that the randomizations do not eliminate those effects. Hence, if $\rho$ dominates $\mu$, then the agent should prefer $\rho$ to $\mu$ independent of his belief about his randomizations.

Axiom (Dominance): For any $A, B \in \mathscr{A}$, if for all $\mu \in \Delta(B)$, there exists $\rho \in \Delta(A)$ such that $\rho$ dominates $\mu$, then $A \succsim B$.

To summarize, the dominance axiom means that the agent should prefer a set $A$ to another set $B$, if for any randomization $\mu$ over $B$, there exists a better randomization

\footnotetext{
${ }^{8}$ Note that under the first three axioms, there exists a unique certainty equivalent $x(f)$ for any act $f \in \mathscr{F}$.
} 
$\rho$ over $A$, regardless of whether he believes that his randomizations eliminate the effects of uncertainty.

THEOREM 1: $\succsim$ satisfies Weak Order, Continuity, Monotonicity, Uncertainty Aversion, Certainty Set Independence, Certainty Strategic Rationality, and Dominance if and only if there exists a pair $(\delta, C)$ of $\delta \in[0,1]$ and $C \subset \Delta(S)$ such that $\succsim$ is represented by a function $U: \mathscr{A} \rightarrow \mathbb{R}$ defined by

$$
U(A)=\max _{\rho \in \Delta(A)}\left[\delta u\left(\sum_{f \in \mathscr{F}} \rho(f) f\right)+(1-\delta) \sum_{f \in \mathscr{F}} \rho(f) u(f)\right],
$$

where $u(f)=\min _{p \in C} \sum_{s \in S} p(s) f(s)$ and $C$ is compact and convex. ${ }^{9}$

We call $\succsim$ an RUA preference relation if $\succsim$ satisfies all the axioms in Theorem 1 . In the online Appendix, we present an axiomatization of an extended representation, in which $u$ is a variational utility function proposed by Maccheroni, Marinacci, and Rustichini (2006).

For an RUA preference relation, both parameters $\delta$ and $C$ are unique whenever the agent is not an expected utility maximizer.

REMARK 1: Suppose that two RUA representations with $(\delta, C)$ and $\left(\delta^{\prime}, C^{\prime}\right)$ represent the same $\succsim$. Then $(i) C=C^{\prime}$, and (ii) if $C$ is not a singleton, then $\delta=\delta^{\prime}$.

In this remark, (i) means that $C$ is uniquely pinned down for the agent. (ii) means that $\delta$ is also uniquely pinned down when the agent is not an expected utility maximizer. When the agent is an expected utility maximizer (i.e., when $C=\{p\}$ ), then the first term and the second term become equal (i.e., $u\left(\sum_{f \in \mathscr{F}} \rho(f) f\right)$ $\left.=\sum_{s \in S} p(s) \sum_{f \in \mathscr{F}} \rho(f) f(s)=\sum_{f \in \mathscr{F}} \rho(f) u(f)\right)$. Therefore, $\delta$ becomes irrelevant to the agent's utility. In this sense, the nonuniqueness of $\delta$ in the case of an expected utility maximizer is unavoidable but not significant.

\section{Characterizations of $\delta$}

In this section, we study how the key parameter $\delta$ describes the agent's preferences. First, we discuss the implication of the two special cases of the RUA representations in which $\delta=1$ and $\delta=0$. After that, we present comparative statics on $\delta$.

The two special cases in which $\delta=1$ and $\delta=0$ imply that the agent believes that his randomization can eliminate the effects of uncertainty completely and that his randomization cannot eliminate those effects at all, respectively. Both implications seem rather extreme. This would suggest that most people would exhibit the generic case in which $\delta \in(0,1)$. Indeed, the next proposition shows that the two special cases are characterized by rather strong axioms, namely the indifference

\footnotetext{
${ }^{9}$ Remember that values of acts are utils. Hence, for all $x \in \mathcal{U}, U(\{(x, \ldots, x)\})=u(x, \ldots, x)=x$.
} 
to randomization axiom (i.e., $A \sim \operatorname{co}(A))$ and the strategic rationality axiom (i.e., $A \succsim B \Rightarrow A \sim A \cup B$ ), respectively.

PROPOSITION 1: Suppose that $\succsim$ is represented by an RUA representation with $(\delta, C)$.

(i) $\succsim$ satisfies the indifference to randomization axiom if and only if $\delta=1$.

(ii) $\succsim$ satisfies the strategic rationality axiom if and only if $\delta=0$.

In what follows, we present comparative statics on $\delta$. We show that both a preference for flexibility (i.e., a preference for a larger choice set) and a preference for randomization are captured by $\delta$. We say that agent 1 has a stronger preference for flexibility than agent 2 if agent 1 prefers a set of acts to an act in the set whenever agent 2 also prefers that set to that act. Formally,

DEFINITION 4: $\succsim_{1}$ is said to exhibit a stronger preference for flexibility than $\succsim_{2}$ if, for every $A \in \mathscr{A}$ and $f \in A$,

$$
A \succsim_{2}\{f\} \Rightarrow A \succsim_{1}\{f\} .
$$

Next, we formalize a comparative attitude across agents toward randomization. Consider an agent whose preference relation $\succsim$ admits an RUA representation with $(\delta, C)$. Then, we define $w(\rho)$ to be the agent's expected utility of randomization $\rho$ when $\rho$ eliminates the effects of uncertainty with probability $\delta$. Formally, for any $\rho \in \Delta(\mathscr{F})$, define $w(\rho)=\delta u\left(\sum_{f \in \mathscr{F}} \rho(f) f\right)+(1-\delta) \sum_{f \in \mathscr{F}} \rho(f) u(f)$, where $u(f)=\min _{p \in C} \sum_{s \in S} p(s) f(s)$.

DEFINITION 5: For an RUA preference relation $\succsim$, we denote by $\Delta_{\succsim}(A)$ the set of

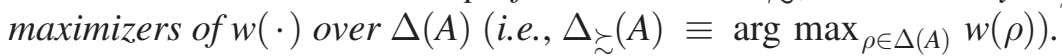

Note that Theorem 1 shows that $\max _{\rho \in \Delta(A)} w(\rho)$ represents $\succsim$. Hence, Theorem 1 guarantees that $\Delta_{\succsim}(A)$ is nonempty. ${ }^{10}$ Note also that $\left(\Delta_{\succsim}(A)\right) \backslash A \neq \emptyset$ means that a nondegenerate randomization is optimal. In other words, the agent has an incentive to randomize his choice. We say that agent 1 has a stronger preference for randomization than agent 2 if agent 1 has an incentive to randomize his choice whenever agent 2 has such an incentive. Formally,

DEFINITION 6: For any RUA preference relations $\succsim_{1}$ and $\succsim_{2}, \succsim_{1}$ is said to exhibit a stronger preference for randomization than $\succsim_{2}$ if for any $A \in \mathscr{A}$,

$$
\left(\Delta_{\succsim_{2}}(A)\right) \backslash A \neq \emptyset \Rightarrow\left(\Delta_{\succsim 1}(A)\right) \backslash A \neq \emptyset .
$$

\footnotetext{
${ }^{10}$ We can directly prove that $\Delta \succ(A) \neq \emptyset$, as follows. Since $S$ is finite and $A$ is compact, $\Delta(A)$ is compact. $\sum_{f \in \mathscr{F}} \rho(f) u(f)$ is continuous in $\rho$. By Berge's maximum theorem, $u\left(\sum_{f \in \mathscr{F}} \rho(f) f\right)$ is also continuous in $\rho$ (see footnote 14 for the detailed argument). Hence, $w(\rho)$ is continuous in $\rho$. Therefore, Weierstrass's theorem shows that $\Delta_{\succsim}(A) \equiv \arg \max _{\rho \in \Delta(A)} w(\rho) \neq \emptyset$.
} 
PROPOSITION 2: Suppose $\succsim_{i}$ is represented by an RUA representation with $\left(\delta_{i}, C_{i}\right)$ for each $i \in\{1,2\}$ and $C_{1}=C_{2}$, where $C_{1}$ is not a singleton. Then, the following conditions are equivalent:

(i) ${ }_{1}$ exhibits a stronger preference for flexibility than $\succsim_{2}$;

(ii) $\delta_{1} \geq \delta_{2}$; and

(iii) $\succsim_{1}$ exhibits a stronger preference for randomization than $\succsim_{2}$.

The result shows that in RUA preference relations, a stronger preference for randomization is equivalent to a stronger preference for flexibility. Moreover, both comparative attitudes are captured by a larger $\delta$.

\section{Application}

In this section, we apply our result to Ellsberg's (1961) experiment: we consider an agent whose preference relation admits an RUA representation. Then, we study how the size of the agent's choice set affects his observable attitude toward uncertainty, namely his uncertainty premium.

Remember that an agent's uncertainty premium is the premium for betting on the ball color drawn from the risky urn: a premium whose magnitude is such as to make him indifferent between (i) betting on the ball color drawn from the risky urn and (ii) betting on the ball color drawn from the uncertain urn. Remember also that the two acts $f^{\text {Red }} \equiv(1,0)$ and $f^{\text {Black }} \equiv(0,1)$ correspond to betting on red and black, respectively, in the uncertain urn. The act $\left(\frac{1}{2}, \frac{1}{2}\right)$ corresponds to betting on a color in the risky urn.

Our framework allows us to define the agent's uncertainty premiums depending on who determines the ball color drawn from the uncertain urn on which the agent bets. When the agent determines it, the uncertainty premium $r^{\text {RedBlack }}$ is defined as follows: $\left\{\left(\frac{1}{2}-r^{\text {RedBlack }}, \frac{1}{2}-r^{\text {RedBlack }}\right)\right\} \sim\left\{f^{\text {Red }}, f^{\text {Black }}\right\}$. Note that, in the left set,

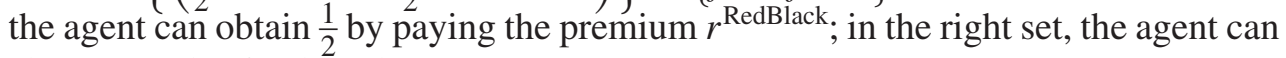
choose a color freely to bet on.

On the other hand, when the experimenter predetermines the color (e.g., red) on which the agent bets, the uncertainty premium $r^{\text {Red }}$ is defined as follows: $\left\{\left(\frac{1}{2}-r^{\text {Red }}, \frac{1}{2}-r^{\text {Red }}\right)\right\} \sim\left\{f^{\text {Red }}\right\}$. We define $r^{\text {Black }}$ in the same way (i.e., $\left.\left\{\left(\frac{1}{2}-r^{\text {Black }}, \frac{1}{2}-r^{\text {Black }}\right)\right\} \sim\left\{f^{\text {Black }}\right\}\right)$.

PROPOSITION 3: Suppose that $\left\{f^{\text {Red }}\right\} \sim\left\{f^{\text {Black }}\right\}$, and $C$ is not a singleton. Then, $\min \left\{r^{\text {Red }}, r^{\text {Black }}\right\}>r^{\text {RedBlack }}$ if and only if $\delta>0$.

This proposition shows that a smaller choice set increases the agent's uncertainty premium as long as $\delta>0$. In other words, the agent behaves as if he were more uncertainty-averse when his choice set is smaller.

In fact, in many experimental studies, an agent is allowed to determine the ball color drawn from the uncertain urn on which he bets. In accordance with 
this convention, we also use $r^{\text {RedBlack }}$ as the agent's uncertainty premium, rather than $r^{\text {Red }}$ or $r^{\text {Black }}$. Consequently, we say that the agent is observationally uncertainty-averse if $r^{\text {RedBlack }}>0$ and that he is observationally uncertainty-neutral if $r^{\text {RedBlack }}=0$. We can calculate $r^{\text {RedBlack }}$ explicitly as follows:

PROPOSITION 4: Suppose that $\left\{f^{\text {Red }}\right\} \sim\left\{f^{\text {Black }}\right\}$. Then,

$$
r^{\text {RedBlack }}=\frac{(1-\delta)|C|}{2}
$$

where $|C|=\max _{p \in C} p($ Red $)-\min _{p \in C} p($ Red $)$. Hence, the agent is observationally uncertainty-averse if and only if $\delta<1$ and $|C|>0$.

This proposition means that the agent's uncertainty premium is determined not only by his set $C$ of priors but also by his subjective belief (i.e., $\delta$ ) that his randomization eliminates the effects of uncertainty. The uncertainty premium is decreasing in $\delta$. Indeed, even if the agent does not have a unique prior (i.e., $|C|>0$ ), he can be observationally uncertainty-neutral (i.e., $r^{\text {RedBlack }}=0$ ) when $\delta=1$.

Given Proposition 4, we can characterize the comparative attitudes across agents. We say that $\succsim_{1}$ is observationally more uncertainty-averse than $\succsim_{2}$ if $r_{1}^{\text {RedBlack }}$ $\geq r_{2}^{\text {RedBlack }}$, where $r_{i}^{\text {RedBlack }}$ is the uncertainty premium for each agent $i \in\{1,2\}$.

COROLLARY 1: Suppose that $\succsim_{i}$ is represented by an RUA representation with $\left(\delta_{i}, C_{i}\right)$ and $\left\{f^{\text {Red }}\right\} \sim_{i}\left\{f^{\text {Black }}\right\}$ for each $i \in\{1,2\}$. Then, $\succsim_{1}$ is observationally more uncertainty-averse than $\succsim_{2}$ if and only if $\left(1-\delta_{1}\right)\left|C_{1}\right| \geq\left(1-\delta_{2}\right)\left|C_{2}\right|$.

This proposition implies that even when agent 1 has a smaller set of priors than agent 2 (i.e., $C_{1} \subset C_{2}$ ), agent 1 could be observationally more uncertainty-averse than agent 2 if $\delta_{1}<\delta_{2}$.

\section{Proofs}

First, we present the sketch for the proof of Theorem 1. Then, we show the proofs of Theorem 1 and Remark 1. Finally, we present the proofs of Propositions 1-4.

\section{A. Sketch of Proof}

In this section, we sketch the proof of sufficiency in Theorem 1. The main difficulty in the proof arises from the fact that we do not assume the indifference to randomization axiom: $A \sim \operatorname{co}(A)$. This axiom has been used by many authors such as Dekel, Lipman, and Rustichini (2001) and Epstein, Marinacci, and Seo (2007).11

\footnotetext{
${ }^{11}$ Dekel, Lipman, and Rustichini (2001) and Epstein, Marinacci, and Seo (2007) impose the indifference to randomization axiom on preferences over sets of lotteries. Epstein, Marinacci, and Seo (2007) propose two representations. For one representation, they impose the indifference to randomization axiom. For the other representation, they do not impose the axiom. Instead, they use richer primitives, that is, preferences over (the second
} 
Since we do not assume this axiom, we cannot focus on convex sets without loss of generality. We overcome this difficulty by investigating sets in a utility space. It turns out that, in the utility space, we can focus on convex sets without loss of generality because of the quasi-concavity of uncertainty-averse preferences.

The outline of the sketch is as follows. First, we focus on sets that contain two indifferent acts (i.e., $\{f, g\}$ such that $f \sim g$ ). Then, we explain how to obtain the desired representation on these particular domains. Finally, we explain how to extend the desired representation into the whole domain.

By a standard argument, there exists a utility function $U: \mathscr{A} \rightarrow \mathbb{R}$ such that $U$ has Gilboa and Schmeidler's (1989) maxmin expected utility representation on $\mathscr{F}$. Consider $\{f, g\}$ such that $f \sim g$. Define

$$
\delta(\{f, g\})=\frac{U(\{f, g\})-U(f)}{\max _{\alpha \in[0,1]} U(\alpha f+(1-\alpha) g)-U(f)} .
$$

By Certainty Set Independence, it can be shown that $\delta$ does not depend on $f$ and $g$. (See Lemma 6 for the proof.) So, we can define $\delta=\delta(\{f, g\})$. By rewriting (3), we obtain $U(\{f, g\})=\max _{\alpha \in[0,1]} \delta U(\alpha f+(1-\alpha) g)+(1-\delta) U(f)$ $=\max _{\alpha \in[0,1]} \delta U(\alpha f+(1-\alpha) g)+(1-\delta)(\alpha U(f)+(1-\alpha) U(g))$. The last equality holds because $U(f)=U(g)$. Define

$$
\mathscr{L}=\{\{f, g\} \mid f \sim g \text { and } \alpha f+(1-\alpha) g \succ f \text { for some } \alpha \in[0,1]\} .
$$

Note that $\delta$ is well defined on $\mathscr{L}$ and that we have obtained the desired representation on $\mathscr{L}$.

Next, we obtain the representation on an arbitrary set $A \in \mathscr{A}$. For this aim, we consider the set that consists of the utilities of randomizations on $A .{ }^{12}$ For any randomization $\rho$, we define $v_{1}(\rho)$ as the utility of $\rho$ when $\rho$ eliminates the effects of uncertainty; and $v_{2}(\rho)$ as the utility of $\rho$ when $\rho$ does not eliminate those effects. Formally, $v_{1}(\rho)=U\left(\sum_{f \in \mathscr{F}} \rho(f) f\right)$; and $v_{2}(\rho)=\sum_{f \in \mathscr{F}} \rho(f) U(f)$. For any set $A \in \mathscr{A}$, define

(5) $A^{*}=\left\{\left(v_{1}, v_{2}\right) \in \mathbb{R}^{2} \mid v_{2}=v_{2}(\rho)\right.$ and $v_{2}(\rho) \leq v_{1} \leq v_{1}(\rho)$ for some $\left.\rho \in \Delta(A)\right\}$.

By Dominance, we can show that if $A^{*}=B^{*}$, then $U(A)=U(B)$. Therefore, without loss of generality, we can focus on this alternative domain $\mathscr{A}^{*}$. (See Lemma 7 for a formal proof.)

This alternative domain has useful properties. It can be shown that $A^{*}=\operatorname{co}\left(\left\{\left(v_{1}(\rho), v_{2}(\rho)\right) \in \mathbb{R}^{2} \mid \rho \in \Delta(A)\right\}\right)$ because of the concavity of $U$ on $\mathscr{F}$. Moreover, Jensen's inequality implies that $v_{1} \geq v_{2}$ for all $\left(v_{1}, v_{2}\right) \in A^{*}$. Hence, $A^{*}$ is a convex set, as described in Figure 3.

stage) lotteries over sets of (the first stage) lotteries. They then assume the independence axiom with respect to the second stage lotteries.

${ }^{12}$ For technical simplicity, $A^{*}$ consists not only of the utilities of randomizations on $A$ but also of the dominated utilities. 


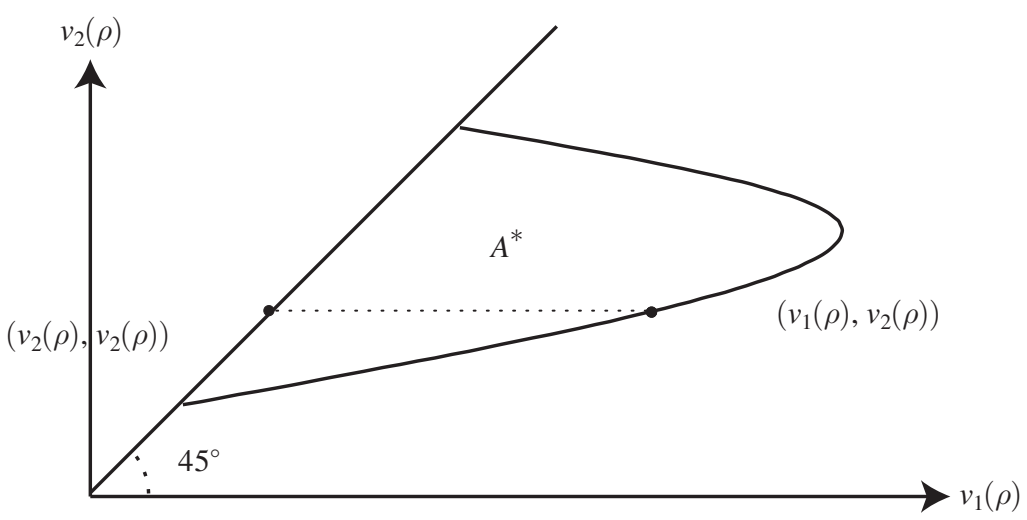

Figure 3. Set $A^{*}$

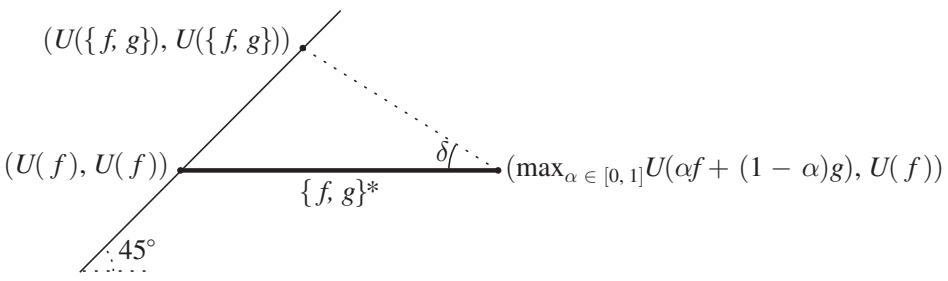

Figure 4. Set $\{f, g\}^{*} \in \mathscr{L}$ AND $\delta$

In particular, if $A \in \mathscr{L}$ (i.e., $A=\{f, g\}$ for some $f, g \in \mathscr{F}$ such that $f \sim g)$, then $A^{*}$ becomes a line segment spanned by $(U(f), U(f))$ and $\left(\max _{\alpha \in[0,1]} U(\alpha f+(1-\alpha) g), U(f)\right)$. Hence, the parameter $\delta$ can be described as the slope of the line connecting $(U(\{f, g\}), U(\{f, g\}))$ and $\left(\max _{\alpha \in[0,1]} U(\alpha f+(1-\alpha) g), U(f)\right)$, as shown in Figure 4. Remember that we have already obtained the desired representation on $\mathscr{L}$ : for any $A \in \mathscr{L}, U(A)$ $=\max _{\rho \in \Delta(A)} \delta U\left(\sum_{f \in \mathscr{F}} \rho(f) f\right)+(1-\delta) \sum_{f \in \mathscr{F}} \rho(f) U(f)=\max _{v \in A^{*}} \delta v_{1}+$ $(1-\delta) v_{2}$.

Finally, we extend the representation to an arbitrary $A \in \mathscr{A}$. For all $\left(v_{1}, v_{2}\right) \in \mathbb{R}^{2}$, define $V\left(v_{1}, v_{2}\right)=\delta v_{1}+(1-\delta) v_{2}$. Let $U^{*}$ be the maximum of $V$ on $A^{*}$ and $\mathbf{v}^{*}$ be its maximizer on $A^{*}$. Let $B, C \in \mathscr{L}$ such that $B^{*}$ and $C^{*}$ are the two line segments respectively described in Figure 5. ${ }^{13}$ Note that $B^{*}$ is the line segment spanned by $\left(v_{2}^{*}, v_{2}^{*}\right)$ and $\mathbf{v}^{*}$. We denote by $\underline{\mathbf{w}}$ and $\overline{\mathbf{w}}$ the two points that span the line segment $C^{*}$. Moreover, the slope of the line connecting $\mathbf{v}^{*}$ and $\left(U^{*}, U^{*}\right)$, and the slope of the line connecting $\underline{\mathbf{w}}$ and $\left(U^{*}, U^{*}\right)$ are the same, namely $\delta$.

\footnotetext{
${ }^{13}$ Existence of such sets will be proved in Lemma 9.
} 


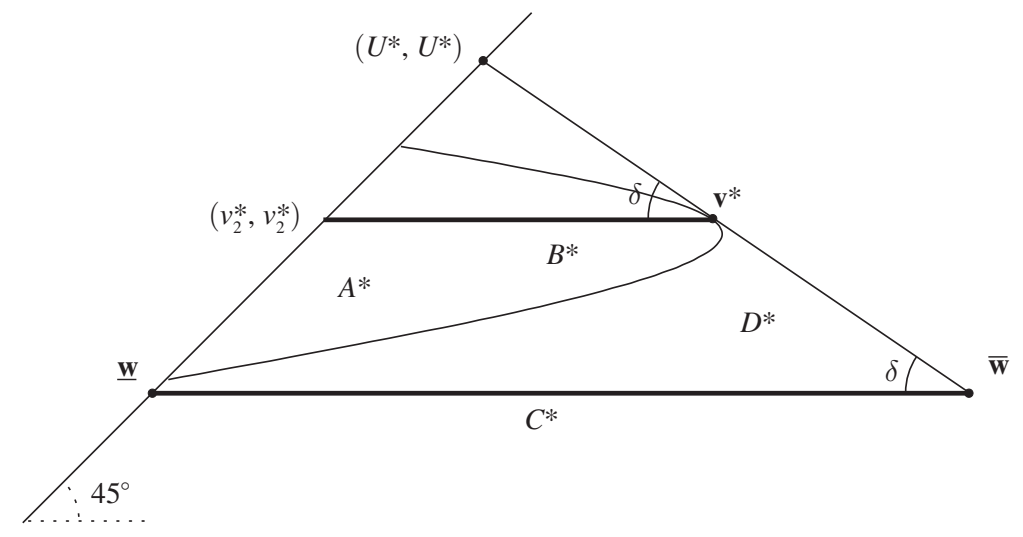

Figure 5. Sets $A^{*}, B^{*}, C^{*}$, AND $D^{*}$

Therefore, by the representation on $\mathscr{L}, \quad U(B)=\max _{\mathbf{v} \in B^{*}} V\left(v_{1}, v_{2}\right)=U^{*}$ $=\max _{\mathbf{v} \in C^{*}} V\left(v_{1}, v_{2}\right)=U(C)$.

Define $D=C \cup\left\{\left(U^{*}, \ldots, U^{*}\right)\right\}$. Since $U\left(U^{*}, \ldots, U^{*}\right)=U^{*}$, Certainty Strategic Rationality shows that $U(D)=U^{*}$. Moreover, $D^{*}=\operatorname{co}\left(C^{*} \cup\left\{\left(U^{*}, U^{*}\right)\right\}\right)$, which is the triangle containing $A^{*}$ described in Figure 5. Since $B^{*} \subset A^{*} \subset D^{*}$, we can show $U(B) \leq U(A) \leq U(D)$ by using Dominance. Since $U(B)=U^{*}=U(D)$, we obtain $U(A)=U^{*} \equiv \max _{\mathbf{v} \in A^{*}} V\left(v_{1}, v_{2}\right)=\max _{\rho \in \Delta(A)} \delta U\left(\sum_{f \in \mathscr{F}} \rho(f) f\right)+$ $(1-\delta) \sum_{f \in \mathscr{F}} \rho(f) U(f)$, where the last equality holds by the definition of $\mathbf{v}$. (See Lemma 9 for a formal proof.)

\section{B. Proof of Sufficiency in Theorem 1}

In this section, we present the proof of sufficiency in Theorem 1. The proof consists of nine lemmas. First, we present the outline of the proof with the statements of the lemmas. After that, we present the proofs of the lemmas. (We put the proofs of several lemmas in the online Appendix.) Based on one's interest, one can read the outline only or the entire section.

We introduce the following notation. We denote a singleton set $\{f\}$ by $f$. We denote constant acts $(x, \ldots, x)$ and $(y, \ldots, y)$ by $x$ and $y$ when there is no danger of confusion. For example, we denote $\alpha f+(1-\alpha)(x, \ldots, x)$ and $\alpha A+(1-\alpha)(x, \ldots, x)$ by $\alpha f+(1-\alpha) x$ and $\alpha A+(1-\alpha) x$, respectively.

Note that Certainty Set Independence implies Gilboa and Schmeidler's (1989) axiom: $f \succsim g \Leftrightarrow \alpha f+(1-\alpha) x \succsim \alpha g+(1-\alpha) x$ for all $f, g \in \mathscr{F}, x \in \mathscr{U}$, and $\alpha \in(0,1)$. Hence, Gilboa and Schmeidler's (1989) theorem shows the next lemma. The proof is in the online Appendix.

LEMMA 1: There exists a nonempty, compact, and convex subset $C$ of $\Delta(S)$ such that $\succsim$ on $\mathscr{F}$ is represented by $u(f)=\min _{p \in C} \sum_{s \in S} p(s) f(s)$.

By Dominance and Continuity, for any $A \in \mathscr{A}$, we can find $f \in \mathscr{F}$ such that $\{f\} \sim A$. Define $U(A)=u(f)$. So by Lemma 1 , we can prove Lemma 2 . The formal proof is in the online Appendix. 
LEMMA 2: There exists a function $U: \mathscr{A} \rightarrow \mathcal{U}$ such that $(i) U(A) \geq U(B) \Leftrightarrow A \succsim B$ and $($ ii $) U(f)=\min _{p \in C} \sum_{s \in S} p(s) f(s)$ for all $f \in \mathscr{F}$.

By Dominance and the concavity of $U$ on $\mathscr{F}$, we obtain the next lemma.

LEMMA 3: $(i)$ For all $\rho \in \Delta(\mathscr{F}), U\left(\sum_{f \in \mathscr{F}} \rho(f) f\right) \geq \sum_{f \in \mathscr{F}} \rho(f) U(f)$; and $(i i)$ $\max _{\rho \in \Delta(A)} U\left(\sum_{f \in \mathscr{F}} \rho(f) f\right) \geq U(A) \geq \max _{\rho \in \Delta(A)} \sum_{f \in \mathscr{F}} \rho(f) U(f)$.

In the next lemma, by using Lemma 3 (ii), we can establish Theorem 1 when $U$ has an expected utility representation on $\mathscr{F}$.

LEMMA 4: Suppose that $U$ has an expected utility representation on $\mathscr{F}$. Then, for any $\delta \in[0,1]$ and $A \in \mathscr{A}, U(A)=\max _{\rho \in \Delta(A)} \delta U\left(\sum_{f \in \mathscr{F}} \rho(f) f\right)+$ $(1-\delta) \sum_{f \in \mathscr{F}} \rho(f) U(f)$.

In the following, we assume that $U$ does not have an expected utility representation on $\mathscr{F}$. Then, by a standard argument, there exist $f^{*}, g^{*} \in \mathscr{F}$ such that $\frac{1}{2} f^{*}+\frac{1}{2} g^{*} \succ f^{*} \sim g^{*}$. (We, henceforth, fix these $f^{*}$ and $g^{*}$.) Define $\mathscr{L}$ by (4) in the sketch of proof. Note that $\left\{f^{*}, g^{*}\right\} \in \mathscr{L}$.

For all $\{f, g\} \in \mathscr{L}$, define $\delta(\{f, g\})$ by (3) in the sketch of proof. By the definition, $\max _{\alpha \in[0,1]} U(\alpha f+(1-\alpha) g)>U(f)$, so the denominator of $\delta(\{f, g\})$ is positive. Therefore, $\delta(\{f, g\})$ is well defined. Moreover, by Lemma 3 (ii), $\max _{\alpha \in[0,1]} U(\alpha f+(1-\alpha) g) \geq U(\{f, g\}) \geq U(f)$, so that $\delta(\{f, g\}) \in[0,1]$.

Since $U$ is a maxmin expected utility function on $\mathscr{F}, U$ is constant linear (i.e., for any $f \in \mathscr{F}, x \in \mathscr{U}$, and $\alpha \in[0,1]$, we have $U(\alpha f+(1-\alpha) x)=\alpha U(f)+(1-\alpha) x)$. Mainly by using this property and Certainty Set Independence, we can prove the next lemma.

LEMMA 5: For all $A \in \mathscr{A}, \alpha \in[0,1]$, and $x \in \mathscr{U}, U(\alpha A+(1-\alpha) x)=\alpha U(A)+$ $(1-\alpha) x$.

By using Lemma 5, in the next lemma, we can show that $\delta$ is constant on $\mathscr{L}$, hence we obtain the desired representation on $\mathscr{L}$.

\section{LEMMA 6:}

There exists $\delta \in[0,1]$ such that $U(\{f, g\})=\max _{\rho \in \Delta(\{f, g\})} \delta U\left(\sum_{f \in \mathscr{F}} \rho(f) f\right)+$ $(1-\delta) \sum_{f \in \mathscr{F}} \rho(f) U(f)$ for all $\{f, g\} \in \mathscr{L}$.

In the following, we extend the representation to the whole domain $\mathscr{A}$. For this purpose, for any $\rho \in \Delta(\mathscr{F})$, define $v_{1}(\rho)=U\left(\sum_{f \in \mathscr{F}} \rho(f) f\right), v_{2}(\rho)=\sum_{f \in \mathscr{F}} \rho(f) U(f)$, and $\mathbf{v}(\rho)=\left(v_{1}(\rho), v_{2}(\rho)\right)$. For any $A \in \mathscr{A}$, define $A^{*}$ by $(5)$ in the sketch of proof. Note that since $\Delta(A)$ is compact and $\mathbf{v}$ is continuous in $\Delta(A), A^{*}$ is compact. ${ }^{14}$

\footnotetext{
${ }^{14}$ Since $S$ is finite and $A$ is compact, $\Delta(A)$ is compact. To see $\mathbf{v}$ is continuous, define $u_{p}(f)=\sum_{s \in S} p(s) f(s)$ for all $p \in C$; and define $\hat{u}_{p}(\mu)=\sum_{f \in \mathscr{F}} \mu(f) u_{p}(f)$ for all $\mu \in \Delta(A)$ and $p \in C$. Note that with the Euclidean metric, $u_{p}(f)$ is continuous with respect to $f$. Moreover, $u_{p}(f)$ is bounded in $\Delta(A)$. Therefore, with the weak convergence
} 
We say that $A^{*}$ dominates $B^{*}$ if for all $\mathbf{v} \in B^{*}$ there exists $\mathbf{u} \in A^{*}$ such that $u_{1} \geq v_{1}$ and $u_{2} \geq v_{2}$. We show two preliminary lemmas before obtaining the desired representation on $\mathscr{A}$.

LEMMA 7: (i) If $A^{*}$ dominates $B^{*}$, then $U(A) \geq U(B)$; (ii) If $A^{*} \supset B^{*}$ then $U(A) \geq U(B) ;$ (iii) For all $\rho \in \Delta(\mathscr{F}), v_{1}(\rho) \geq v_{2}(\rho)$; and (iv) $\max _{\mathbf{v} \in A^{*}} v_{1} \geq$ $U(A) \geq \max _{\mathbf{v} \in A^{*}} v_{2}$.

Note that Lemma 7 (ii) shows that if $A^{*}=B^{*}$, then $U(A)=U(B)$.

Remember that we fixed $f^{*}, g^{*} \in \mathscr{F}$ such that $\frac{1}{2} f^{*}+\frac{1}{2} g^{*} \succ f^{*} \sim g^{*}$. Let $\alpha^{*} \in \arg \max _{\alpha \in[0,1]} U\left(\alpha f^{*}+(1-\alpha) g^{*}\right)$. For simplicity, assume $U\left(f^{*}\right)=0$ and $U\left(\alpha^{*} f^{*}+\left(1-\alpha^{*}\right) g^{*}\right)=1$ without loss of generality. ${ }^{15}$ For all $\beta \in[0,1]$, define $f[\beta]=\beta f^{*}+(1-\beta) x\left(f^{*}\right)$ and $g[\beta]=\beta g^{*}+(1-\beta) x\left(g^{*}\right)$. (We use the notation $f[\beta]$ instead of $f(\beta)$ to avoid confusion because $f(s) \in \mathbb{R}$ for each $s \in S$.) Given the definitions, by using Lemma 6, we obtain (i) in the next lemma. (ii) follows from Certainty Strategic Rationality.

LEMMA 8: (i) For any $\beta \in(0,1],\{f[\beta], g[\beta]\}^{*}=\operatorname{co}(\{(0,0),(\beta, 0)\})$ and $U(\{f[\beta], g[\beta]\})=\delta \beta ;$ (ii) For any $\beta \in[0,1]$ and $x \in \mathcal{U},\{f[\beta], g[\beta],(x, \ldots, x)\}^{*}$ $=\operatorname{co}(\{(0,0),(\beta, 0),(x, x)\})$.

By using Lemma 7 and 8 , we establish the desired representation on $\mathscr{A}$ in the next lemma.

LEMMA 9:

For all $A \in \mathscr{A}, U(A)=\max _{\rho \in \Delta(A)} \delta U\left(\sum_{f \in \mathscr{F}} \rho(f) f\right)+(1-\delta) \sum_{f \in \mathscr{F}} \rho(f) U(f)$.

In the following, we provide the proofs of main lemmas. We omit the proofs of Lemmas 1 and 2. The proofs are standard and in the online Appendix.

\section{PROOF OF LEMMA 3:}

For any $\rho \in \Delta(\mathscr{F}), \quad U\left(\sum_{f \in \mathscr{F}} \rho(f) f\right) \geq \sum_{f \in \mathscr{F}} \rho(f) U(f)$, where the inequality holds by Jensen's inequality. Hence, (i) holds. To show (ii), fix $A \in \mathscr{A}$. Let $\bar{f} \in \arg \max _{f \in \operatorname{co}(A)} U(f)$ and $\underline{f} \in \arg \max _{f \in A} U(f)$. Hence, by (i), for any $\rho \in \Delta(A), \quad U(\bar{f}) \geq U\left(\sum_{f \in \mathscr{F}} \rho(\bar{f}) f\right) \geq \sum_{f \in \mathscr{F}} \rho(f) U(f)$. Therefore, $\{(U(\bar{f})$, $U(\bar{f}))\}$ dominates $A$ and $A$ dominates $\{(U(\underline{f}), U(\underline{f}))\}$. Therefore, by Dominance, $U(\bar{f}) \geq U(A) \geq U(\underline{f})$.

topology, by definition $\hat{u}_{p}(\mu)$ is continuous with respect to $\mu$ in $\Delta(A)$. Finally, since $C$ is compact under the product topology, Berge's maximum theorem shows that $v_{1}(\mu) \equiv \min _{p \in C} \hat{u}_{p}(\mu)$ is continuous with respect to $\mu$ in $\Delta(A)$. In the same way, we can show $v_{2}(\mu)$ is continuous in $\Delta(A)$. Therefore, $\mathbf{v}(\mu)=\left(v_{1}(\mu), v_{2}(\mu)\right)$ is continuous with respect to $\mu$ in $\Delta(A)$.

${ }^{15}$ This assumption is only for notational simplicity. Without this assumption, the proof goes through in the same way. However, we need to write $U(f[\beta])$ and $U\left(\alpha^{*} f[\beta]+\left(1+\alpha^{*}\right) g[\beta]\right)$, instead of 0 and $\beta$, respectively. 


\section{PROOF OF LEMMA 4:}

Suppose that $U$ has an expected utility representation on $\mathscr{F}$. Then, there exists $p \in \Delta(S)$ such that for any $f \in \mathscr{F}, U(f)=\sum_{s \in S} f(s) p(s)$. Then $U\left(\sum_{f \in \mathscr{F}} \rho(f) f\right)$ $=\sum_{f \in \mathscr{F}} \rho(f) U(f)$. By Lemma 3 (ii),

$$
U(A)=\max _{\rho \in \Delta(A)} U\left(\sum_{f \in \mathscr{F}} \rho(f) f\right)=\max _{\rho \in \Delta(A)} \sum_{f \in \mathscr{F}} \rho(f) U(f) .
$$

\section{PROOF OF LEMMA 5:}

Fix $A \in \mathscr{A}$. By using Dominance and Continuity, we can find $f \in \mathscr{F}$ such that $A \sim f$. Fix $\alpha \in[0,1]$ and $x \in \mathcal{U}$. Hence, by Certainty Set Independence, $\alpha A+(1-\alpha) x \sim \alpha f+(1-\alpha) x$. Therefore, $U(\alpha A+(1-\alpha) x)=U(\alpha f+(1-\alpha) x)$ $=\alpha U(f)+(1-\alpha) x=\alpha U(A)+(1-\alpha) x$, where the second equality holds by the constant linearity of $U$.

\section{PROOF OF LEMMA 6:}

Choose any $\{f, g\},\left\{f^{\prime}, g^{\prime}\right\} \in \mathscr{L}$ to show $\delta(\{f, g\})=\delta\left(\left\{f^{\prime}, g^{\prime}\right\}\right)$. Let $\alpha^{*}$ $\in \arg \max _{\alpha \in[0,1]} U(\alpha f+(1-\alpha) g)$ and $\beta^{*} \in \arg \max _{\beta \in[0,1]} U\left(\beta f^{\prime}+(1-\beta) g^{\prime}\right)$. We show the lemma in the following two exclusive and inclusive cases.

Case 1: First, consider the case where $U(f)=U\left(f^{\prime}\right)$. Define $x=U(f)$. Without loss of generality assume that $U\left(\beta^{*} f^{\prime}+\left(1-\beta^{*}\right) g^{\prime}\right) \leq U\left(\alpha^{*} f+\left(1-\alpha^{*}\right) g\right)$. Define

$$
\gamma=\frac{U\left(\beta^{*} f^{\prime}+\left(1-\beta^{*}\right) g^{\prime}\right)-x}{U\left(\alpha^{*} f+\left(1-\alpha^{*}\right) g\right)-x} .
$$

Then, $\gamma \in[0,1]$. Define $\hat{f}=\gamma f+(1-\gamma) x$ and $\hat{g}=\gamma g+(1-\gamma) x$. We show that $\delta(\{\hat{f}, \hat{g}\})=\delta(\{f, g\})$ and $\delta\left(\left\{f^{\prime}, g^{\prime}\right\}\right)=\delta(\{\hat{f}, \hat{g}\})$ in the following two steps.

Step 1: $\delta(\{\hat{f}, \hat{g}\})=\delta(\{f, g\})$.

\section{PROOF OF STEP 1:}

By Lemma $5, U(\{\hat{f}, \hat{g}\})=\gamma U(\{f, g\})+(1-\gamma) x$. By the constant linearity of $U, \alpha^{*} \in \arg \max _{\alpha \in[0,1]} U(\alpha \hat{f}+(1-\alpha) \hat{g}) \cdot 16$ Moreover, $U\left(\alpha^{*} \hat{f}+\left(1-\alpha^{*}\right) \hat{g}\right)$ $=\gamma U\left(\alpha^{*} f+\left(1-\alpha^{*}\right) g\right)+(1-\gamma) x$ and $U(\hat{f})=\gamma U(f)+(1-\gamma) x$. It follows that

$$
\begin{aligned}
\delta(\{\hat{f}, \hat{g}\}) & =\frac{U(\{\hat{f}, \hat{g}\})-U(\hat{f})}{U\left(\alpha^{*} \hat{f}+\left(1-\alpha^{*}\right) \hat{g}\right)-U(\hat{f})} \\
& =\frac{\gamma(U(\{f, g\})-U(f))}{\gamma\left(U\left(\alpha^{*} f+\left(1-\alpha^{*}\right) g\right)-U(f)\right)}=\delta(\{f, g\}) .
\end{aligned}
$$

Step 2: $\delta\left(\left\{f^{\prime}, g^{\prime}\right\}\right)=\delta(\{\hat{f}, \hat{g}\})$.

\footnotetext{
${ }^{16}$ For all $\alpha, \beta \in[0,1], x \in \mathcal{U}, U(\alpha(\beta f+(1-\beta) x)+(1-\alpha)(\beta g+(1-\beta) x))=\beta U(\alpha f+(1-\alpha) g)+$ $(1-\beta) x$.
} 


\section{PROOF OF STEP 2:}

First, we show $\left\{f^{\prime}, g^{\prime}\right\} \sim\{\hat{f}, \hat{g}\}$ by using Dominance. By the definition of $\gamma$ and the constant linearity of $U, U\left(\alpha^{*} \hat{f}+\left(1-\alpha^{*}\right) \hat{g}\right)=\gamma U\left(\alpha^{*} f+\left(1-\alpha^{*}\right) g\right)+$ $(1-\gamma) x=U\left(\beta^{*} f^{\prime}+\left(1-\beta^{*}\right) g^{\prime}\right)$. By the constant linearity of $U, U(\hat{f})=\gamma U(f)+$ $(1-\gamma) x=U\left(f^{\prime}\right)$.

Now choose any $\rho \in \Delta(\{\hat{f}, \hat{g}\})$ to show that $\beta^{*} f^{\prime} \oplus\left(1-\beta^{*}\right) g^{\prime}$ dominates $\rho$. Note that $U\left(\beta^{*} f^{\prime}+\left(1-\beta^{*}\right) g^{\prime}\right)=U\left(\alpha^{*} \hat{f}+\left(1-\alpha^{*}\right) \hat{g}\right) \geq U(\rho(\hat{f}) \hat{f}+$ $\rho(\hat{g}) \hat{g})$ and $\beta^{*} U\left(f^{\prime}\right)+\left(1-\beta^{*}\right) U\left(g^{\prime}\right)=U\left(f^{\prime}\right)=U(\hat{f})=\rho(\hat{f}) U(\hat{f})+\rho(\hat{g}) U(\hat{g})$. Hence, Dominance shows $\left\{f^{\prime}, g^{\prime}\right\} \succsim\{\hat{f}, \hat{g}\}$. In the same way, we can obtain $\{\hat{f}, \hat{g}\} \succsim\left\{f^{\prime}, g^{\prime}\right\}$. It follows that $\left\{f^{\prime}, g^{\prime}\right\} \sim\{\hat{f}, \hat{g}\}$, so that $U\left(\left\{f^{\prime}, g^{\prime}\right\}\right)=U(\{\hat{f}, \hat{g}\})$. Therefore,

$$
\begin{aligned}
\delta\left(\left\{f^{\prime}, g^{\prime}\right\}\right) & =\frac{U\left(\left\{f^{\prime}, g^{\prime}\right\}\right)-U\left(f^{\prime}\right)}{U\left(\alpha^{*} f^{\prime}+\left(1-\alpha^{*}\right) g^{\prime}\right)-U\left(f^{\prime}\right)} \\
& =\frac{U(\{\hat{f}, \hat{g}\})-U(\hat{f})}{U\left(\beta^{*} \hat{f}+\left(1-\beta^{*}\right) \hat{g}\right)-U(\hat{f})}=\delta(\{\hat{f}, \hat{g}\}) .
\end{aligned}
$$

By Step 1 and 2, we obtain $\delta(\{f, g\})=\delta\left(\left\{f^{\prime}, g^{\prime}\right\}\right)$.

Case 2: Next consider the case where $U(f) \neq U\left(f^{\prime}\right)$. Without loss of generality assume that $U(f)>U\left(f^{\prime}\right)$. There exists $x \in \mathscr{U}$ such that $U(f)>x>U\left(f^{\prime}\right)$. Then, there exist $\gamma, \gamma^{\prime} \in(0,1)$ such that $\gamma U(f)+(1-\gamma) x=\gamma^{\prime} U\left(f^{\prime}\right)+\left(1-\gamma^{\prime}\right) x$. Then by Case $1, \delta(\{\gamma f+(1-\gamma) x, \quad \gamma g+(1-\gamma) x\})=\delta\left(\left\{\gamma^{\prime} f^{\prime}+\left(1-\gamma^{\prime}\right) x\right.\right.$, $\left.\left.\gamma^{\prime} g^{\prime}+\left(1-\gamma^{\prime}\right) x\right\}\right)$. As in Step 1 of Case 1, we can show that $\delta(\{f, g\})$ $=\delta(\{\gamma f+(1-\gamma) x, \gamma g+(1-\gamma) x\})$. In the same way, we obtain $\delta\left(\left\{f^{\prime}, g^{\prime}\right\}\right)$ $=\delta\left(\left\{\gamma^{\prime} f^{\prime}+\left(1-\gamma^{\prime}\right) x, \gamma^{\prime} g^{\prime}+\left(1-\gamma^{\prime}\right) x\right\}\right)$. Hence, $\delta(\{f, g\})=\delta\left(\left\{f^{\prime}, g^{\prime}\right\}\right)$.

By the arguments in Case 1 and 2, we can define $\delta=\delta(\{f, g\})$ for any $\{f, g\} \in \mathscr{L}$. Finally, to show the representation, choose any $\{f, g\} \in \mathscr{L}$. By the definition of $\delta$,

$$
\delta=\frac{U(\{f, g\})-U(f)}{U\left(\alpha^{*} f+\left(1-\alpha^{*}\right) g\right)-U(f)} .
$$

By rewriting the equation, we obtain $U(\{f, g\})=\delta U\left(\alpha^{*} f+\left(1-\alpha^{*}\right) g\right)+$ $(1-\delta) U(f)$. Note that $U\left(\alpha^{*} f+\left(1-\alpha^{*}\right) g\right)=\max _{\rho \in \Delta(\{f, g\})} U\left(\sum_{h \in \mathscr{F}} \rho(h) h\right)$ and that $U(f)=\sum_{h \in \mathscr{F}} \rho(h) U(h)$ for any $\rho \in \Delta(\{f, g\})$. It follows that $U(\{f, g\})$ $=\max _{\rho \in \Delta(\{f, g\})} \delta U\left(\sum_{h \in \mathscr{F}} \rho(h) h\right)+(1-\delta) \sum_{h \in \mathscr{F}} \rho(h) U(h)$.

\section{PROOF OF LEMMA 7:}

To show (i), fix $A^{*}$ and $B^{*}$ such that $A^{*}$ dominates $B^{*}$. Then, for any $\rho \in \Delta(B)$, there exists $\mu \in \Delta(A)$ such that $v_{1}(\rho) \leq v_{1}(\mu)$ and $v_{2}(\rho) \leq v_{2}(\mu)$. Therefore, by Dominance, $A \succsim B$, so that $U(A) \geq U(B)$. Moreover, if $A^{*} \supset B^{*}$, then $A^{*}$ dominates $B^{*}$, so that $U(A) \geq U(B)$. Hence, (i) and (ii) hold. Lemma 3 and the definitions of $v_{1}$ and $v_{2}$ show (iii) and (iv). 
PROOF OF LEMMA 8:

By the definitions, for all $\beta \in(0,1], \quad U(f[\beta])=0=U(g[\beta])$ and $\max _{\alpha \in[0,1]} U(\alpha f[\beta]+(1-\alpha) g[\beta])=\max _{\alpha \in[0,1]} \beta U\left(\alpha f^{*}+(1-\alpha) g^{*}\right)+$ $(1-\beta) U\left(f^{*}\right)=\beta U\left(\alpha^{*} f^{*}+\left(1-\alpha^{*}\right) g^{*}\right)=\beta$. Note that $\{f[\beta], g[\beta]\} \in \mathscr{L}$. By Lemma $6, U(\{f[\beta], g[\beta]\})=\delta U\left(\alpha^{*} f[\beta]+\left(1-\alpha^{*}\right) g[\beta]\right)+(1-\delta)\left(\alpha^{*} U(f[\beta])+\right.$ $\left.\left(1-\alpha^{*}\right) U(g[\beta])\right)=\delta \beta$.

It is easy to see that $\{f[\beta], g[\beta]\}^{*} \subset \operatorname{co}(\{(0,0),(\beta, 0)\})$. To show the converse, choose, $\mathbf{v}^{\prime} \in \operatorname{co}(\{(0,0),(\beta, 0)\})$. Then, $U(g[\beta])=0 \leq v_{1}^{\prime} \leq \beta=U\left(\alpha^{*} f[\beta]+\right.$ $\left.\left(1-\alpha^{*}\right) g[\beta]\right)$ and $v_{2}^{\prime}=0$. Since $U(\alpha f[\beta]+(1-\alpha) g[\beta])$ is continuous in $\alpha$, by the intermediate value theorem, there exists $\alpha \in\left[0, \alpha^{*}\right]$ such that $U(\alpha f[\beta]+(1-\alpha) g[\beta])=v_{1}^{\prime}$ and $\alpha U(f[\beta])+(1-\alpha) U(g[\beta])=v_{2}^{\prime}, \quad$ so that $\mathbf{v}^{\prime}=\mathbf{v}(\alpha f[\beta] \oplus(1-\alpha) g[\beta])$. Therefore, $\quad \mathbf{v}^{\prime} \in\{f[\beta], g[\beta]\}^{*}$. It follows that $\operatorname{co}(\{(0,0),(\beta, 0)\}) \subset\{f[\beta], g[\beta]\}^{*}$. This completes the proof of $(\mathrm{i})$.

To show (ii), choose any $\beta \in(0,1]$ and $x \in \mathcal{U}$. It is easy to see that $\{f[\beta], g[\beta],(x, \ldots, x)\}^{*} \subset \operatorname{co}(\{(0,0),(\beta, 0),(x, x)\})$. To show the converse, choose any $\mathbf{v}^{\prime} \in \operatorname{co}(\{(0,0),(\beta, 0),(x, x)\})$. If $\mathbf{v}^{\prime} \in \operatorname{co}(\{(0,0),(\beta, 0)\})$, then by the argument in $(\mathrm{i}), \mathbf{v}^{\prime} \in\{f[\beta], g[\beta]\}^{*} \subset\{f[\beta], g[\beta],(x, \ldots, x)\}^{*}$. If $\mathbf{v}^{\prime} \notin \operatorname{co}(\{(0,0),(\beta, 0)\})$, then there exists $\mathbf{v}^{\prime \prime} \in \operatorname{co}(\{(0,0),(\beta, 0)\})$ and $\gamma \in[0,1]$ such that $\mathbf{v}^{\prime}=\gamma \mathbf{v}^{\prime \prime}+$ $(1-\gamma)(x, x)$. By the argument in (i), there exists $\alpha \in[0,1]$ such that $\mathbf{v}(\alpha f[\beta] \oplus(1-\alpha) g[\beta])=\mathbf{v}^{\prime \prime}$. By the constant linearity of $u$, we obtain $\mathbf{v}(\gamma \alpha f[\beta] \oplus \gamma(1-\alpha) g[\beta] \oplus(1-\gamma)(x, \ldots, x))=\gamma \mathbf{v}(\alpha f[\beta] \oplus(1-\alpha) g[\beta])+$ $(1-\gamma)(x, x)=\gamma \mathbf{v}^{\prime \prime}+(1-\gamma)(x, x)=\mathbf{v}^{\prime}$. Therefore $\mathbf{v}^{\prime} \in\{f[\beta], g[\beta],(x, \ldots, x)\}^{*}$.

\section{PROOF OF LEMMA 9:}

Choose any $A \in \mathscr{A}$. We show the lemma in the following two exclusive and inclusive cases.

Case 1: First we consider the case in which $\delta=0$. For all $\alpha \in(0,1)$, define $A_{\alpha}=\alpha A+(1-\alpha)\left(\frac{1}{2}, \ldots, \frac{1}{2}\right)$. By Lemma 7 (iii), for all $\mathbf{u} \in A^{*}, u_{1} \geq u_{2}$. Moreover, since $A^{*}$ is bounded, there exists a positive number $\alpha$ such that $A_{\alpha}^{*} \subset$ $\operatorname{co}(\{(0,0),(1,0),(1,1)\})$. Let $\mathbf{v}^{*}=\arg \max _{\mathbf{v} \in A_{\alpha}^{*}} v_{2}$. (Such $\mathbf{v}^{*}$ exists because $v_{2}$ is continuous and $A_{\alpha}^{*}$ is compact.) Since $A_{\alpha}^{*} \subset \operatorname{co}(\{(0,0),(1,0),(1,1)\})$, it follows that $v_{2}^{*}<1$. Define $B=v_{2}^{*}(1, \ldots, 1)+\left(1-v_{2}^{*}\right)\{f[1], g[1]\}$. By Lemma 5, $U(B)$ $=v_{2}^{*}+\left(1-v_{2}^{*}\right) U(\{f[1], g[1]\})$. By Lemma 8 (i) and $\delta=0, U(\{f[1], g[1]\})=0$. Hence, $U(B)=v_{2}^{*}$. By Lemma 7 (iv), $U(B)=v_{2}^{*} \equiv \max _{\mathbf{v} \in A_{\alpha}^{*}} v_{2} \leq U\left(A_{\alpha}\right)$.

Next we show that $U(B) \geq U\left(A_{\alpha}\right)$. For all $\mathbf{v} \in A_{\alpha}^{*}, v_{1} \leq 1$ and $v_{2} \leq v_{2}^{*}$ because $A_{\alpha}^{*} \subset \operatorname{co}(\{(0,0),(1,0),(1,1)\})$ and $v_{2}^{*}=\max _{\mathbf{v} \in A_{\alpha}^{*}} v_{2}$. Note that $B^{*}=v_{2}^{*}(1,1)+$ $\left(1-v_{2}^{*}\right) \operatorname{co}(\{(0,0),(1,0)\})=\operatorname{co}\left(\left\{\mathbf{v}^{*},\left(1, v_{2}^{*}\right)\right\}\right)$. Therefore, $B^{*}$ dominates $A_{\alpha}^{*}$ as shown in Figure 6. Hence, by Lemma $7(\mathrm{i}), U(B) \geq U\left(A_{\alpha}\right)$.

It follows that $U\left(A_{\alpha}\right)=U(B)=\max _{\mathbf{v} \in A_{\alpha}^{*}} v_{2}=\alpha \max _{\mathbf{v} \in A^{*}} v_{2}+(1-\alpha) \frac{1}{2}$. By Lemma 5, $U\left(A_{\alpha}\right)=\alpha U(A)+(1-\alpha) \frac{1}{2}$. Hence, we obtain $\alpha \max _{\mathbf{v} \in A^{*}} v_{2}+(1-\alpha) \frac{1}{2}$ $=\alpha U(A)+(1-\alpha) \frac{1}{2}$. Therefore, $U(A)=\max _{\mathbf{v} \in A^{*}} v_{2}=\max _{\rho \in \Delta(A)} \sum_{f \in \mathscr{F}} \rho(f) U(f)$.

Case 2: Next we consider the case in which $\delta>0$. Choose a positive number $\varepsilon$ such that $\varepsilon<\delta$. For all $\alpha \in(0,1)$, define $A_{\alpha}=\alpha A+(1-\alpha)(\varepsilon, \varepsilon)$. Then, 


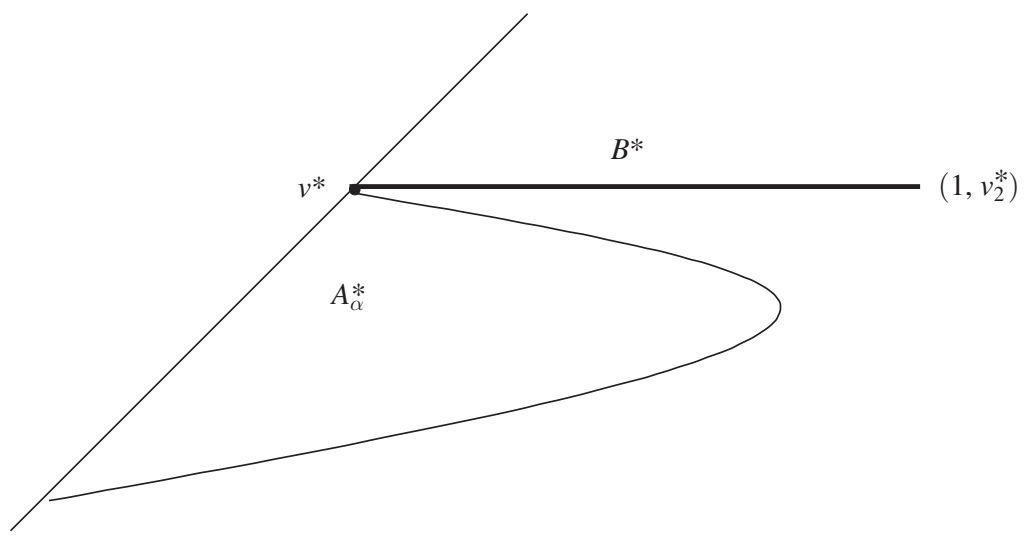

Figure 6. SETS $A^{*}$ AND $B^{*}$ IN CASE 1

$\max _{\mathbf{v} \in A_{\alpha}^{*}} \delta v_{1}+(1-\delta) v_{2}=\alpha\left(\max _{\mathbf{v} \in A^{*}} \delta v_{1}+(1-\delta) v_{2}\right)+(1-\alpha) \varepsilon$. Since $(\mathrm{i}) A^{*}$ is bounded, (ii) $\varepsilon<\delta$, and (iii) $v_{1} \geq v_{2}$ for all $\mathbf{v} \in A^{*}$, there exists a positive number $\alpha$ such that $A_{\alpha}^{*} \subset \operatorname{co}(\{(0,0),(1,0),(1,1)\})$ and $\max _{\mathbf{v} \in A_{\alpha}^{*}} \delta v_{1}+(1-\delta) v_{2}<$ $\delta$. Note that $U\left(A_{\alpha}\right)=\alpha U(A)+(1-\alpha) \varepsilon$ and $\max _{\mathbf{v} \in A_{\alpha}^{*}} \delta v_{1}+(1-\delta) v_{2}$ $=\alpha\left(\max _{\mathbf{v} \in A^{*}} \delta v_{1}+(1-\delta) v_{2}\right)+(1-\alpha) \varepsilon$. Hence to show the lemma, it suffices to prove $U\left(A_{\alpha}\right)=\max _{\mathbf{v} \in A_{\alpha}^{*}} \delta v_{1}+(1-\delta) v_{2}$. Define $U^{*}=\max _{\mathbf{v} \in A_{\alpha}^{*}} \delta v_{1}+(1-\delta) v_{2}$. Then, $U^{*}<\delta$. We show that $U\left(A_{\alpha}\right) \geq U^{*}$ and $U\left(A_{\alpha}\right) \leq U^{*}$ in the following two steps.

Step 1: $U\left(A_{\alpha}\right) \geq U^{*}$.

\section{PROOF OF STEP 1:}

Let $\mathbf{v}^{*} \in \arg \max _{\mathbf{v} \in A_{\alpha}^{*}} \delta v_{1}+(1-\delta) v_{2}$. (Such $\mathbf{v}^{*}$ exists because $\delta v_{1}+(1-\delta) v_{2}$ is continuous and $A_{\alpha}^{*}$ is compact.) By Lemma 7 (iii), $v_{1}^{*} \geq v_{2}^{*}$. Define $B$ $=\frac{v_{2}^{*}}{v_{1}^{*}}\left(v_{1}^{*}, \ldots, v_{1}^{*}\right)+\left(1-\frac{v_{2}^{*}}{v_{1}^{*}}\right)\left\{f\left[v_{1}^{*}\right], g\left[v_{1}^{*}\right]\right\} . \quad$ By $\quad$ Lemma $\quad 5, \quad U(B)=\frac{v_{2}^{*}}{v_{1}^{*}} v_{1}^{*}+$ $\left(1-\frac{v_{2}^{*}}{v_{1}^{*}}\right) U\left(\left\{f\left[v_{1}^{*}\right], g\left[v_{1}^{*}\right]\right\}\right)$. By Lemma $8(\mathrm{i}), U\left(\left\{f\left[v_{1}^{*}\right], g\left[v_{1}^{*}\right]\right\}\right)=\delta v_{1}^{*}$. Therefore, $U(B)=v_{2}^{*}+\left(v_{1}^{*}-v_{2}^{*}\right) \delta=\delta v_{1}^{*}+(1-\delta) v_{2}^{*}=U^{*}$. Moreover, $B^{*}=\frac{v_{2}^{*}}{v_{1}^{*}}\left(v_{1}^{*}, v_{1}^{*}\right)+$ $\left(1-\frac{v_{2}^{*}}{v_{1}^{*}}\right) \operatorname{co}\left(\left\{(0,0),\left(v_{1}^{*}, 0\right)\right\}\right)=\operatorname{co}\left(\left\{\left(v_{2}^{*}, v_{2}^{*}\right), \mathbf{v}^{*}\right\}\right)$. Since $\mathbf{v}^{*} \in A_{\alpha}^{*}$ and $v_{1}^{*} \geq v_{2}^{*}$, we obtain $A_{\alpha}^{*} \supset B^{*}$. Hence, Lemma 7 (ii) shows $U\left(A_{\alpha}\right) \geq U(B)$. It follows that $U\left(A_{\alpha}\right) \geq U^{*}$.

\section{Step 2: $U\left(A_{\alpha}\right) \leq U^{*}$.}

\section{PROOF OF STEP 2:}

Remember that $U^{*}<\delta$. Define $D=\left\{f\left[\frac{U^{*}}{\delta}\right], g\left[\frac{U^{*}}{\delta}\right],\left(U^{*}, \ldots, U^{*}\right)\right\}$. Then, by Lemma 8 (ii), $D^{*}=\operatorname{co}\left(\left\{(0,0),\left(\frac{U^{*}}{\delta}, 0\right),\left(U^{*}, U^{*}\right)\right\}\right)$. By the definitions of $U^{*}$ and $\mathbf{v}^{*}$, we obtain $A_{\alpha}^{*} \subset D^{*}$ (as shown in Figure 7). Therefore, Lemma 7 (ii) shows $U\left(A_{\alpha}\right) \leq U(D)$. Hence, to show $U\left(A_{\alpha}\right) \leq U^{*}$, it suffices to show $U^{*}=U(D)$. Since 


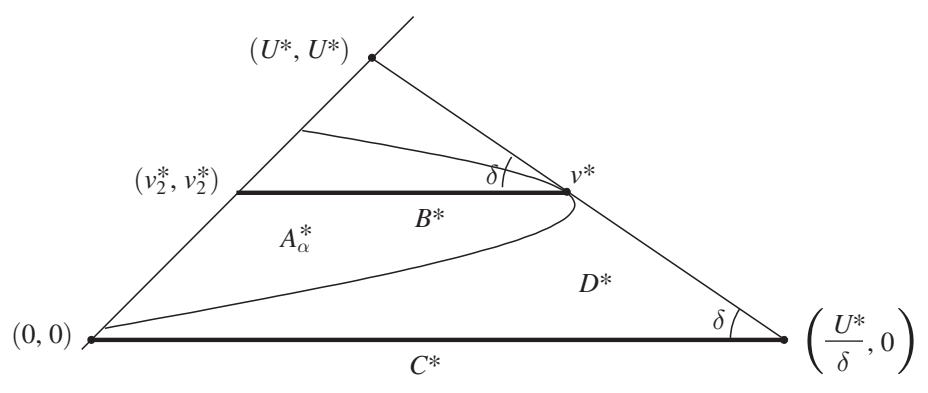

Figure 7. Sets $A^{*}, B^{*}, C^{*}$, And $D^{*}$ In Case 2

$\delta>U^{*}$, then $\left(\frac{U^{*}}{\delta}\right)<1$. Define $C=\left\{f\left[\frac{U^{*}}{\delta}\right], g\left[\frac{U^{*}}{\delta}\right]\right\}$. By Lemma $8(\mathrm{i}), U(C)=U^{*}$. It follows that $\left(U^{*}, \ldots, U^{*}\right) \sim\left\{f\left[\frac{U^{*}}{\delta}\right], g\left[\frac{U^{*}}{\delta}\right]\right\}$. By Certainty Strategic Rationality, $\left(U^{*}, \ldots, U^{*}\right) \sim\left\{f\left[\frac{U^{*}}{\delta}\right], g\left[\frac{U^{*}}{\delta}\right],\left(U^{*}, \ldots, U^{*}\right)\right\} \equiv D$. Hence, $U^{*}=U(D)$.

\section{Proof of Necessity in Theorem 1}

We show that an RUA representation satisfies the two key axioms, Certainty Strategic Rationality and Dominance. An RUA representation trivially satisfies the other axioms. For all $\rho \in \Delta(\mathscr{F})$, define $w(\rho)=\delta u\left(\sum_{f \in \mathscr{F}} \rho(f) f\right)+$ $(1-\delta) \sum_{f \in \mathscr{F}} \rho(f) u(f)$. Note that $U(A)=\max _{\rho \in \Delta(A)} w(\rho)$.

To show that $\succsim$ satisfies Certainty Strategic Rationality, assume $(x, \ldots, x) \succsim B$. Then, $u(x, \ldots, x) \geq U(B)=\max _{\rho \in \Delta(B)} w(\rho)$. Hence, $u(x, \ldots, x) \geq w(\rho)$ for all $\rho \in \Delta(B)$. Note that $w(\alpha \rho \oplus(1-\alpha) x)=\alpha w(\rho)+(1-\alpha) w(x, \ldots, x)$ for all $\rho \in \Delta(B)$ and $\alpha \in[0,1]$. Therefore, $u(x, \ldots, x) \geq w(\mu)$ for all $\mu \in \Delta((x, \ldots, x) \cup B)$. Hence, $u(x, \ldots, x) \geq \max _{\mu \in \Delta((x, \ldots, x) \cup B)} w(\mu) \equiv U((x, \ldots, x) \cup B)$, so that $(x, \ldots, x)$ $\succsim(x, \ldots, x) \cup B$.

To show that $\succsim$ satisfies Dominance, fix $A, B \in \mathscr{A}$ and suppose that for any $\mu \in \Delta(B)$, there exists $\rho \in \Delta(A)$ such that $\rho$ dominates $\mu$. That is, $u\left(\sum_{f \in \mathscr{F}} \rho(f) f\right) \geq u\left(\sum_{f \in \mathscr{F}} \mu(f) f\right)$ and $\sum_{f \in \mathscr{F}} \rho(f) u(f) \geq \sum_{f \in \mathscr{F}} \mu(f) u(f)$. Since $\delta \in[0,1], \quad$ then $w(\rho) \equiv \delta u\left(\sum_{f \in \mathscr{F}} \rho(f) f\right)+(1-\delta) \sum_{f \in \mathscr{F}} \rho(f) u(f)$ $\geq \delta u\left(\sum_{f \in \mathscr{F}} \mu(f) f\right)+(1-\delta) \sum_{f \in \mathscr{F}} \mu(f) u(f) \equiv w(\mu)$. Hence, $U(A)$ $\equiv \max _{\rho \in \Delta(A)} w(\rho) \geq \max _{\mu \in \Delta(B)} w(\mu) \equiv U(B)$, so that $A \succsim B$.

\section{Proof of Remark 1 (Uniqueness)}

The uniqueness of $C$ (i.e., $C=C^{\prime}$ ) follows from Gilboa and Schmeidler (1989). Let $u$ and $u^{\prime}$ be maxmin expected utilities defined with $C$ and $C^{\prime}$ respectively. Then, by normalization that $u(x, \ldots, x)=x=u^{\prime}(x, \ldots, x)$, we obtain $u=u^{\prime}$.

Suppose that $C$ is not a singleton. Then, there exist $f^{*}, g^{*} \in \mathscr{F}$ such that $\frac{1}{2} f^{*}+$ $\frac{1}{2} g^{*} \succ f^{*} \sim g^{*}$. Let $\alpha^{*} \in \arg \max _{\alpha \in[0,1]} U\left(\alpha f^{*}+(1-\alpha) g^{*}\right)$. Define $x=\delta u\left(\alpha^{*} f^{*}+\right.$ 
$\left.\left(1-\alpha^{*}\right) g^{*}\right)+(1-\delta) u\left(f^{*}\right)$. Then, $U\left(\left\{f^{*}, g^{*}\right\}\right)=x$, so that $\left\{f^{*}, g^{*}\right\} \sim(x, \ldots, x)$. Hence, $\left.x=u^{\prime}(x, \ldots, x)=\delta^{\prime} u^{\prime}\left(\alpha^{*} f^{*}+\left(1-\alpha^{*}\right) g^{*}\right)+\left(1-\delta^{\prime}\right) u^{\prime}\left(f^{*}\right\}\right)$. Since $u=u^{\prime}$, we obtain

$$
\delta=\frac{x-u\left(f^{*}\right)}{u\left(\alpha^{*} f^{*}+\left(1-\alpha^{*}\right) g^{*}\right)-u\left(f^{*}\right)}=\frac{x-u^{\prime}\left(f^{*}\right)}{u^{\prime}\left(\alpha^{*} f^{*}+\left(1-\alpha^{*}\right) g^{*}\right)-u^{\prime}\left(f^{*}\right)}=\delta^{\prime} .
$$

\section{E. Proofs of Propositions 1-4}

First, we present a reminder for a basic fact: When $C$ is not a singleton, there exist $f^{*}, g^{*} \in \mathscr{F}$, and $\alpha^{*} \in[0,1]$ such that $\alpha^{*} f^{*}+\left(1-\alpha^{*}\right) g^{*} \succsim \alpha f^{*}+(1-\alpha) g^{*}$ for all $\alpha \in[0,1]$ and $\alpha^{*} f^{*}+\left(1-\alpha^{*}\right) g^{*} \succ f^{*} \sim g^{*}$. Hence, $U\left(\left\{f^{*}, g^{*}\right\}\right)=\delta u\left(\alpha^{*} f^{*}+\right.$ $\left.\left(1-\alpha^{*}\right) g^{*}\right)+(1-\delta) u\left(f^{*}\right)$.

\section{PROOF OF PROPOSITION 1:}

First, we show (i). It is easy to see that if $\delta=1$ then $\succsim$ exhibits Indifference to Randomization. To show the converse, let $A=\left\{f^{*}, g^{*}\right\}$. By Indifference to Randomization, $A \sim \operatorname{co}(A)$. Therefore, $\delta u\left(\alpha^{*} f^{*}+\left(1-\alpha^{*}\right) g^{*}\right)+(1-\delta) u\left(f^{*}\right)$ $=U(A)=U(\operatorname{co}(A))=\max _{h \in \operatorname{co}(A)} u(h)=u\left(\alpha^{*} f^{*}+\left(1-\alpha^{*}\right) g^{*}\right)$, so that $\delta=1$. Next, we show (ii). It is easy to see that if $\delta=0$, then $\succsim$ exhibits Strategic Rationality. To show the converse, note that by Strategic Rationality, $A \sim\left\{f^{*}\right\}$. Therefore, $\delta u\left(\alpha^{*} f^{*}+\left(1-\alpha^{*}\right) g^{*}\right)+(1-\delta) u\left(f^{*}\right)=U(A)=U\left(\left\{f^{*}\right\}\right)=u\left(f^{*}\right)$, so that $\delta=0$.

\section{PROOF OF PROPOSITION 2:}

Fix two RUA preference relations $\left\{\succsim_{i}\right\}_{i=1,2}$ represented by $\left\{\left(\delta_{i}, C_{i}\right)\right\}_{i=1,2}$, where $C_{1}=C_{2}$.

\section{Step 1: (i) $\Leftrightarrow($ ii $)$.}

\section{PROOF OF STEP 1:}

First, we show that (i) implies (ii). Suppose that $\succsim_{1}$ exhibits a stronger preference for flexibility than $\succsim_{2}$. Define $u(f)=\min _{p \in C} \sum_{s \in S} p(s) f(s)$. In the following, we show $\delta_{1} \geq \delta_{2}$. Let $U_{1}$ and $U_{2}$ be the RUA representations with $\left(\delta_{1}, C\right)$ and $\left(\delta_{2}, C\right)$, respectively. Define $x=\delta_{2} u\left(\alpha^{*} f^{*}+\left(1-\alpha^{*}\right) g^{*}\right)+$ $\left(1-\delta_{2}\right) u\left(f^{*}\right)$. Since $U_{2}\left(\left\{f^{*}, g^{*},(x, \ldots, x)\right\}\right)=\delta_{2} u\left(\alpha^{*} f^{*}+\left(1-\alpha^{*}\right) g^{*}\right)+$ $\left(1-\delta_{2}\right) u\left(f^{*}\right)=x$, then $\left\{f^{*}, g^{*},(x, \ldots, x)\right\} \sim_{2}(x, \ldots, x)$. Since $\succsim_{1}$ exhibits a stronger preference for flexibility than $\succsim_{2}$, then $\left\{f^{*}, g^{*},(x, \ldots, x)\right\} \succsim_{1}(x, \ldots, x)$. Hence, $U_{1}\left(\left\{f^{*}, g^{*},(x, \ldots, x)\right\}\right)=\delta_{1} u\left(\alpha^{*} f^{*}+\left(1-\alpha^{*}\right) g^{*}\right)+\left(1-\delta_{1}\right) u\left(f^{*}\right) \geq x$. Therefore,

$$
\delta_{1} \geq \frac{x-u\left(f^{*}\right)}{u\left(\alpha^{*} f^{*}+\left(1-\alpha^{*}\right) g^{*}\right)-u\left(f^{*}\right)}=\delta_{2}
$$


Second, we show that (ii) implies (i). Suppose that $\delta_{1} \geq \delta_{2}$ and $C_{1}=C_{2}$. Define $u(f)=\min _{p \in C_{1}} \sum_{s \in S} p(s) f(s)$. Fix any $A \in \mathscr{A}$ and $g \in A$ such that $A \succsim_{2} g$ to show $A \succsim_{1} g$. Then, for any $\rho \in \Delta_{\succsim_{2}}(A)$,

$$
\begin{array}{rlrl}
U_{1}(A) & \geq \delta_{1} u\left(\sum_{f \in \mathscr{F}} \rho(f) f\right)+\left(1-\delta_{1}\right) \sum_{f \in \mathscr{F}} \rho(f) u(f) & \\
& \geq \delta_{2} u\left(\sum_{f \in \mathscr{F}} \rho(f) f\right)+\left(1-\delta_{2}\right) \sum_{f \in \mathscr{F}} \rho(f) u(f) & \left(\because \delta_{1} \geq \delta_{2}\right) \\
& =U_{2}(A) & \left(\because \rho \in \Delta_{\succsim_{2}}(A)\right) \\
\geq u(g) . & \left(\because A \succsim_{2} g\right)
\end{array}
$$

Step 2: (ii) $\Leftrightarrow$ (iii) .

\section{PROOF OF STEP 2:}

Note that by definition, for all $i \in\{1,2\}, \Delta_{\succsim_{i}}(A)=\arg \max _{\rho \in \Delta(A)} w_{i}(\rho)$.

First, we show that (ii) implies (iii). Suppose that $\delta_{1} \geq \delta_{2}$. Choose $A \in \mathscr{A}$ such that $\left(\Delta_{\succsim 2}(A)\right) \backslash A \neq \emptyset$. Choose any $\rho^{*} \in\left(\Delta_{\succsim 2}(A)\right) \backslash A$. Then, $w_{2}\left(\rho^{*}\right) \geq w_{2}(f)$ for any $f \in \widetilde{A}$. Therefore,

$$
\begin{aligned}
w_{1}\left(\rho^{*}\right) & =\delta_{1} u\left(\sum_{f \in \mathscr{F}} \rho^{*}(f) f\right)+\left(1-\delta_{1}\right) \sum_{f \in \mathscr{F}} \rho^{*}(f) u(f) \\
& \geq \delta_{2} u\left(\sum_{f \in \mathscr{F}} \rho^{*}(f) f\right)+\left(1-\delta_{2}\right) \sum_{f \in \mathscr{F}} \rho^{*}(f) u(f) \quad\left(\because \delta_{1} \geq \delta_{2}\right) \\
& =w_{2}\left(\rho^{*}\right) .
\end{aligned}
$$

So, we obtain $w_{1}\left(\rho^{*}\right) \geq w_{2}\left(\rho^{*}\right)$. Since $w_{2}\left(\rho^{*}\right) \geq w_{2}(f)$ and $w_{2}(f)=u(f)=w_{1}(f)$ for any $f \in A$, it follows that $w_{1}\left(\rho^{*}\right) \geq w_{1}(f)$ for any $f \in A$. Hence, if there exists $f \in A$ such that $f \in \Delta_{\succsim 1}(A)$, then $\rho^{*} \in\left(\Delta_{\succsim 1}(A)\right) \backslash A \neq \emptyset$. If there exists no $f \in A$ such that $f \in \Delta_{\succsim 1}(A)$, then $\left(\Delta_{\succsim 1}(A)\right) \backslash A=\Delta_{\succsim_{1}}(A) \neq \emptyset$. (Remember that $\Delta_{\succsim 1}(A) \neq \emptyset$.) In either case, $\left(\Delta_{\succsim 1}(A)\right) \backslash \widetilde{A} \neq \emptyset$.

Second, we show that (iii) implies (ii). Suppose that $\succsim_{1}$ exhibits a stronger preference for randomization than $\succsim_{2}$. We show $\delta_{1} \geq \delta_{2}$. Define $x=w_{2}\left(\alpha^{*} f^{*} \oplus\left(1-\alpha^{*}\right) g^{*}\right)$ and $A=\left\{f^{*}, g^{*},(x, \ldots, x)\right\}$. Then, $\alpha^{*} f^{*} \oplus\left(1-\alpha^{*}\right) g^{*}$ $\in\left(\Delta_{\succsim_{2}}(A)\right) \backslash A \neq \emptyset$. By way of contradiction suppose that $\delta_{1}<\delta_{2}$, then

$$
\begin{aligned}
x & =w_{2}\left(\alpha^{*} f^{*} \oplus\left(1-\alpha^{*}\right) g^{*}\right) \\
& =\delta_{2} u\left(\alpha^{*} f^{*}+\left(1-\alpha^{*}\right) g^{*}\right)+\left(1-\delta_{2}\right) u\left(f^{*}\right) \\
& >\delta_{1} u\left(\alpha^{*} f^{*}+\left(1-\alpha^{*}\right) g^{*}\right)+\left(1-\delta_{1}\right) u\left(f^{*}\right) \quad\left(\because \delta_{2}>\delta_{1}\right) \\
& =w_{1}\left(\alpha^{*} f^{*} \oplus\left(1-\alpha^{*}\right) g^{*}\right) .
\end{aligned}
$$


By the constant linearity of $u, \Delta_{\succsim 1}(A) \equiv \arg \max _{\rho \in \Delta(A)} w(\rho)=\{(x, \ldots, x)\}$, so that $\Delta_{\succsim 1}(A) \backslash A=\emptyset$. This contradicts that $\succsim_{1}$ exhibits a stronger preference for randomization than $\succsim_{2}$.

\section{PROOF OF PROPOSITIONS 3 AND 4:}

First, we show Proposition 4. If $u\left(f^{\text {Red }}\right)=u\left(f^{\text {Black }}\right)$, then $\min _{p \in C} p($ Red $)$ $=\min _{p \in C} p$ (Black). Hence, $U\left(\left\{f^{\text {Red }}, f^{\text {Black }}\right\}\right)=\max _{\alpha \in[0,1]} w\left(\alpha f^{\text {Red }} \oplus(1-\alpha)\right.$ $\left.\times f^{\text {Black }}\right)=w\left(\frac{1}{2} f^{\text {Red }} \oplus \frac{1}{2} f^{\text {Black }}\right)=\delta \frac{1}{2}+(1-\delta)\left(\frac{1}{2} \min _{p \in C} p(\right.$ Red $)+$ $\frac{1}{2} \min _{p \in C} p($ Black $\left.)\right)=\frac{1}{2}(1-(1-\delta)|C|)$, where the last equality holds because $\min _{p \in C} p($ Black $)=1-\max _{p \in C} p($ Red $)$. Since $\frac{1}{2}-r^{\text {RedBlack }}=U\left(\left\{f^{\text {Red }}, f^{\text {Black }}\right\}\right)$, by the definition of $r^{\text {RedBlack }}$, then $r^{\text {RedBlack }}=\frac{1}{2}(1-\delta)|C|$.

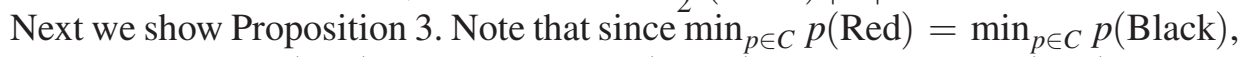
we obtain $\max _{p \in C} p($ Red $)=1-\min _{p \in C} p($ Black $)=1-\min _{p \in C} p($ Red $)$. Therefore, by the definition of $|C|$, we obtain $\frac{1}{2}(1-\delta)|C|=\frac{1}{2}(1-\delta)(1-$ $\left.2 \min _{p \in C} p(\operatorname{Red})\right)=(1-\delta)\left(\frac{1}{2}-\min _{p \in C} p(\operatorname{Red})\right)^{2}$.

By the definition of $r^{\text {Red }}, \frac{1}{2}-r^{\text {Red }}=u\left(f^{\text {Red }}\right)$. Since $u\left(f^{\text {Red }}\right)=\min _{p \in C} p($ Red $)$, we obtain $r^{\text {Red }}=\frac{1}{2}-\min _{p \in C} p($ Red $)$. Since $u\left(f^{\text {Red }}\right)=u\left(f^{\text {Black }}\right)$, then $r^{\text {Red }}=r^{\text {Black }}$. Hence, $\min \left\{r^{\text {Red }}, r^{\text {Black }}\right\}>r^{\text {RedBlack }}$ if and only if $\frac{1}{2}-\min _{p \in C} p($ Red $)>(1-\delta)$ $\times\left(\frac{1}{2}-\min _{p \in C} p(\operatorname{Red})\right)$ if and only if $\delta>0$.

\section{REFERENCES}

Anscombe, Francis J., and Robert J. Aumann. 1963. “A Definition of Subjective Probability." Annals of Mathematical Statistics 34 (1): 199-205.

Battigalli, Pierpaolo, Simone Cerreia-Vioglio, Fabio Maccheroni, and Massimo Marinacci. 2013. "Mixed Extensions of Decision Problems under Uncertainty." Bocconi University IGIER Working Paper 485.

Dekel, Eddie, Barton L. Lipman, and Aldo Rustichini. 2001. "Representing Preferences with a Unique Subjective State Space.” Econometrica 69 (4): 891-934.

Eichberger, Jürgen, Simon Grant, and David Kelsey. 2014. "Randomization and Dynamic Consistency." Unpublished.

-Elssberg, Daniel. 1961. "Risk, Ambiguity, and the Savage Axioms." Quarterly Journal of Economics 75 (4): 643-69.

Epstein, Larry G., Massimo Marinacci, and Kyoungwon Seo. 2007. "Coarse Contingencies and Ambiguity." Theoretical Economics 2 (4): 355-94.

Gilboa, Itzhak. 2009. Theory of Decision under Uncertainty. New York: Cambridge University Press.

Gilboa, Itzhak, and David Schmeidler. 1989. “Maxmin Expected Utility with Non-unique Prior.” Journal of Mathematical Economics 18 (2): 141-53.

Gul, Faruk, and Wolfgang Pesendorfer. 2006. "Random Expected Utility." Econometrica 74 (1): 121-46.

-Kreps, David M. 1979. “A Representation Theorem for 'Preference for Flexibility'.” Econometrica 47 (3): 565-77.

Kuzmics, Christoph. 2013. "A Rational Ambiguity Averse Person Will Never Display Her Ambiguity Aversion." Unpublished.

Maccheroni, Fabio, Massimo Marinacci, and Aldo Rustichini. 2006. "Ambiguity Aversion, Robustness, and the Variational Representation of Preferences." Econometrica 74 (6): 1447-98.

-Manzini, Paola, and Marco Mariotti. 2014. "Stochastic Choice and Consideration Sets." Econometrica 82 (3): 1153-76.

-Raiffa, Howard. 1961. "Risk, Ambiguity, and the Savage Axioms: Comment." Quarterly Journal of Economics 75: 690-94.

-Saito, Kota. 2013. "Social Preferences under Risk: Equality of Opportunity versus Equality of Outcome." American Economic Review 103 (7): 3084-101.

Savage, Leonard J. 1954. The Foundations of Statistics. New York: John Wiley \& Sons. 